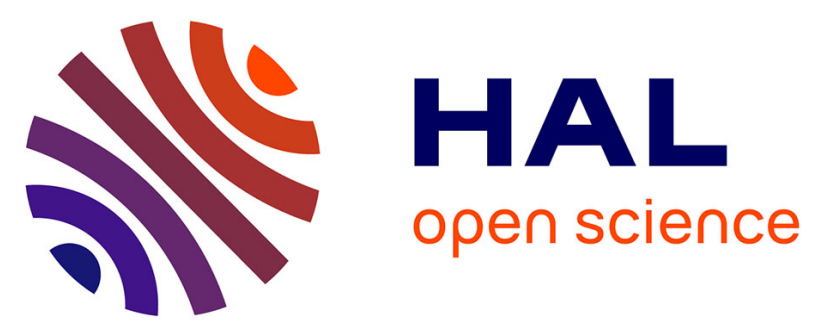

\title{
Resolving the stratification discrepancy of turbulent natural convection in differentially heated air-filled cavities. Part III: a full convection-conduction-surface radiation coupling
}

Shihe Xin, Jacques Salat, Patrice Joubert, Anne Sergent, François Penot, Patrick Le Quéré

\section{To cite this version:}

Shihe Xin, Jacques Salat, Patrice Joubert, Anne Sergent, François Penot, et al.. Resolving the stratification discrepancy of turbulent natural convection in differentially heated air-filled cavities. Part III: a full convection-conduction-surface radiation coupling. International Journal of Heat and Fluid Flow, 2013, 42, pp.33-48. 10.1016/j.ijheatfluidflow.2013.01.021 . hal-00958721

\section{HAL Id: hal-00958721 \\ https://hal.science/hal-00958721}

Submitted on 10 Apr 2014

HAL is a multi-disciplinary open access archive for the deposit and dissemination of scientific research documents, whether they are published or not. The documents may come from teaching and research institutions in France or abroad, or from public or private research centers.
L'archive ouverte pluridisciplinaire HAL, est destinée au dépôt et à la diffusion de documents scientifiques de niveau recherche, publiés ou non, émanant des établissements d'enseignement et de recherche français ou étrangers, des laboratoires publics ou privés. 


\title{
Resolving the stratification discrepancy of turbulent natural convection in differentially heated air-filled cavities. Part III: a full convection-conduction-surface radiation coupling.
}

Shihe Xin ${ }^{1}$, Jacques Salat ${ }^{2}$, Patrice Joubert ${ }^{3}$, Anne Sergent ${ }^{4,5}$, François Penot ${ }^{2}$ and Patrick Le Quéré ${ }^{4}$

${ }^{1}$ INSA-Lyon, UMR5008, CETHIL, 20, av. A. Einstein, F-69621 Villeurbanne Cedex

2 LET-ENSMA, UMR6608, BP 40109, F-86961 Futuroscope cedex

${ }^{3}$ LEPTAB, Université de La Rochelle, Av. M. Crépeau, F-17042 La Rochelle cedex 1

${ }^{4}$ LIMSI-CNRS, BP 133, F-91403 Orsay Cedex

${ }^{5}$ UPMC Univ Paris 06, F-75005, Paris cedex

Tel : 334724370 77, Fax : 334724388 11, e-mail : shihe.xin@insa-lyon.fr

\begin{abstract}
The present study concerns an air-filled differentially heated cavity of $1 \mathrm{~m} \times 0.32 \mathrm{~m} \times 1 \mathrm{~m}$ (width $\times$ depth $\times$ height) subject to a temperature difference of $15 \mathrm{~K}$ and is motivated by understanding the persistent discrepancy observed on thermal stratification in the cavity core between numerical and experimental results. An improved experiment with enhanced metrology was set up and experimental data have been obtained along with the characteristics of the surfaces and materials used in the set-up. Especially experimental temperature distributions on the passive walls have been introduced in numerical simulations and this is one way for numerical simulation to predict correctly the experimental results. By means of DNS using spectral methods, heat conduction in the insulating material is first coupled with natural convection in cavity. As heat conduction influences only temperature distribution on the top and bottom surfaces and in the near wall regions, surface radiation is added to the coupling of natural convection with heat conduction. The polycarbonate front and rear walls of the cavity, which are almost black surfaces for low temperature radiation, and also other low emissivity walls affect strongly temperature distribution in the cavity: thermal stratification is considerably weakened by surface radiation. Good agreement between numerical simulations and experiments is observed on both time-averaged fields and turbulent statistics. Treating the full conduction-convection-radiation coupling allowed to confirm that experimental wall temperatures resulted from the coupled phenomena and this is another way to predict correctly the experimental results in the cavity.
\end{abstract}




\section{Introduction}

Three-dimensional numerical simulations of natural convection flows in an air filled cavity have been performed for the idealized boundary conditions (adiabatic horizontal and vertical passive walls) and also for realistic boundary conditions (measured temperature) on the horizontal walls [22]. Despite the tremendous efforts, no improvement on agreement between experimental and numerical results has been observed: the discrepancy, already observed on the thermal stratification and the temperature profiles on the cavity horizontal walls between two-dimensional computations and experimental measurements, still remains important.

In Parts I and II [25, 24], efforts have been made to understand the reasons for the discrepancy and improve the agreement between numerical and experimental studies: apart from the Intermediate Realistic Cavity (IRC), periodically realistic and fully realistic thermal boundary conditions have been introduced and the corresponding configurations, namely PRC (Periodically Realistic Cavity) and FRC (Fully Realistic Cavity), have been investigated by using LES approach. PRC configuration was chosen in order to have compatible approaches with those used by Peng and Davidson [19]. In a FRC, thermal conditions on the 6 cavity walls are of Dirichlet type and provided by experimental measurements. This ensures that when working on the same physical problem (the same boundary value problem) both experimental and numerical studies provide similar results which are in good agreement. Therefore, understanding the reason why the discrepancy persists and where the realistic thermal boundary conditions come from is more than ever a current topic.

Three reasons for the discrepancy or the fully realistic thermal boundary conditions are possible: non-Boussinesq effects, the coupling between air convection and heat conduction in insulating polyurethane foam and the coupling of air convection with both heat conduction in polyurethane foam and radiation between cavity internal surfaces. As in the experiment $\Delta T=15 \mathrm{~K}$ is within the validity limit of Boussinesq assumption established for air by Gray and Giorgini [10], it is still doubtful that non-Boussinesq effects influence more than $50 \%$ the thermal stratification in the cavity core, especially when the experimental measurements showed no sign of important centro-symmetry breaking [21]. We can therefore exclude, at least at the current stage, non-Boussinesq effects from consideration.

In this study we present briefly experimental studies performed and investigate numerically the last two possible reasons. It is known that air has low thermal conductivity and it is difficult to achieve adiabatic conditions for an air-filled cavity as polyurethane foam has $\lambda_{f}=0.027 \mathrm{~W} /(\mathrm{m} \mathrm{K})$ to be compared with $\lambda=0.025 \mathrm{~W} /(\mathrm{m} \mathrm{K})$ of dry air. The coupling between convection in air and heat conduction in polyurethane foam needs thus to be taken into account. This is supported by the recent work of Omri and Galanis [18] 
who investigated the experimental case of Tian and Karayiannis [28] and observed significant effect on numerical results when considering heat conduction in the horizontal walls.

As far as surface radiation is concerned, its importance is supported by the following facts:

- Net radiative flux is not small compared with convective heat flux. The cavity active walls are made of polished aluminium. Typical emissivity of polished aluminium surface is about 0.1. Net radiative flux exchanged between these walls is proportional to $\epsilon \sigma\left(T_{h}^{4}-T_{c}^{4}\right) \sim 4 \epsilon \sigma T_{0}^{3}\left(T_{h}-T_{c}\right)$ where $\epsilon=0.1, \sigma=5.67 \times$ $10^{-8} \mathrm{~W} /\left(\mathrm{m}^{2} \mathrm{~K}^{4}\right)$ is Stefan-Boltzmann constant, $T_{0}=\left(T_{h}+T_{c}\right) / 2=295.5 \mathrm{~K}$ is the average temperature and $T_{h}$ and $T_{c}$ are respectively the hot and cold temperatures. When scaled with conduction flux, $\lambda \Delta T / H(\lambda=0.025 \mathrm{~W} /(\mathrm{m} \mathrm{K})$, air thermal conductivity and $H=1 \mathrm{~m}$, cavity height), dimensionless net radiative flux ( $\left.4 \epsilon \sigma H T_{0}^{3} / \lambda\right)$ is equal to about 24 , roughly $40 \%$ of convective heat transfer. The above rough estimation indicates that not only surface radiation can not be neglected in natural convection in the cavity considered but also the estimated magnitude of radiative net flux is important in terms of heat transfer.

- Surface radiation changes dramatically thermal boundary conditions. Adiabatic conditions imply the balance between convective flux and net radiative flux, this means that temperature gradient is no longer equal to zero at the adiabatic walls. Interface conditions at polyurethane foam surfaces will result from the balance of conduction in polyurethane foam, convection in air and surface radiation.

- The front and rear walls are almost black bodies. The cavity surfaces in the experiment are at low temperatures and the corresponding surface radiation is in infra-red. The front and rear walls are made of polycarbonate, they are opaque for infra-red and have thus strong emissivities.

- Emissivities of the front and rear walls do influence the thermal stratification in the cavity core [21]. With decreasing the emissivities (from 0.97 to 0.1 ) of the front and rear walls, the stratification measured for depth/height aspect ratio of 0.32 increased from 0.375 to 0.44 . This is certainly not enough to explain the difference between numerical and experimental results but it does suggest that surface radiation is important in the cavity and should be investigated in order to understand its effects.

We are thus motivated by investigating surface radiation and its influence on heat transfer and flow structures in the cavity and numerical methods have been developed to enable numerical studies of the coupling between surface radiation and natural convection. 
Although there are in the literature numerous references for natural convection in cavities, works on interactions between surface radiation and natural convection are rare. As far as rectangular cavities are considered, participating medium is investigated by Lauriat [14], Chang et al. [6], Fusegi and Farouk [8], Yücel et al [35], Han and Baek [12], Kassemi and Naraghi [13], and Colomer et al [7], partitioned cavities are considered by Chang et al. [6], Han and Baek [12] and Mezrhab and Bchir [17] and finally transparent medium in cavities of simple geometry is investigated by Behnia et al [4], Balaji and Venkateshan [2, 3], Kassemi and Naraghi [13], Akiyama and Chong [1], Velusamy et al [30] and Colomer et al [7]. Among studies performed for transparent medium in cavities of simple geometry, only Colomer et al [7] and Borjini et al [5] performed threedimensional simulations and Velusamy et al [30], Sharma et al [26] and Xamán et al [32] investigated interactions of turbulent natural convection with surface radiation by using two-dimensional $k-\epsilon$ modelling. There are obviously difficulties in finding test cases in order to validate numerical procedures developed for investigating three-dimensional turbulent natural convection interacting with surface radiation. Even in two-dimensional laminar cases, there is no benchmark problem on interactions of natural convection with surface radiation for the purpose of code validation. Therefore in the present 3D work only basic tests have been done for numerical procedures used. Note nevertheless that the approach used has been tested and validated previously in 2D cases [31].

As has been observed in Part II [24], LES using the temperature fields experimentally measured on the passive walls yielded numerical results in good agreement with the experimental data. Our last motivation is to understand the physical phenomena which result in the experimentally observed temperature distributions on the passive walls.

This paper is organized as follows: the next section concerns the physical problem which is followed by the mathematical formulation and numerical methods. Numerical results will be presented and discussed before giving the concluding remarks.

\section{Physical problem}

We are interested in an air-filled cavity of $1 \mathrm{~m}$ wide in $x$ direction (width $W=1 \mathrm{~m}$ ), $0.32 \mathrm{~m}$ deep in $y$ direction (depth $D=0.32 \mathrm{~m}$ ) and $1 \mathrm{~m}$ high in $z$ direction (height $H=1 \mathrm{~m})$. The corresponding experimental facility displayed in Figure 1 is an airfilled cavity of $1 \mathrm{~m} \times 1 \mathrm{~m} \times 1 \mathrm{~m}$. The cavity floor and ceiling are made of polyurethane foams of $H_{f}=100 \mathrm{~mm}$ thick $\left(\lambda_{f}=0.027 \mathrm{~W} /(\mathrm{m} \mathrm{K})\right.$ and $\left.\kappa_{f}=3.04 \times 10^{-7} \mathrm{~m}^{2} / \mathrm{s}\right)$ and they are covered by an aluminium foil of $70 \mu \mathrm{m}$ thick with low emissivity. The two active (isothermal) walls are realized with $10 \mathrm{~mm}$ polished aluminium plates. In order to prevent heat loss through the vertical passive walls, the whole cavity is divided in the depth $(y)$ direction into three cavities of equal size by transparent polycarbonate sheets of $1 \mathrm{~mm}$ thick. Measurements are done only in the central cavity and the present 
experimental set-up is an improved version of the cavity studied by Mergui and Penot [16] with enhanced temperature and velocity metrology.

In order to stay within the limit of Boussinesq assumption, temperature difference between the hot and cold walls, $\Delta T=T_{h}-T_{c}$, is kept to be equal to $15 \mathrm{~K}$ ( $T_{h}=303 \mathrm{~K}$ and $\left.T_{c}=288 \mathrm{~K}\right)$ around the ambient temperature which is equal to the mean temperature, $T_{0}=\left(T_{h}+T_{c}\right) / 2=295.5 \mathrm{~K}$. In terms of non-dimensional parameters, natural convection flows in the cavity depend on the geometrical aspect ratios $\left(A_{x}=W / H=1, A_{y}=\right.$ $D / H=0.32$ and $A_{f}=H_{f} / H=0.1$ ), Prandtl number $\operatorname{Pr}=\nu / \kappa=0.71$ and Rayleigh number $R a=\left(g \beta \Delta T H^{3}\right) /(\nu \kappa)=1.5 \times 10^{9}$ where $\beta=0.003 \mathrm{~K}^{-1}$ (thermal expansion coefficient), $\nu=1.5 \times 10^{-5} \mathrm{~m}^{2} / \mathrm{s}$ (molecular viscosity) and $\kappa=2.1126 \times 10^{-5} \mathrm{~m}^{2} / \mathrm{s}$ (thermal diffusivity) are calculated at the mean temperature $T_{0}$.

In this work, surface radiation between the cavity internal surfaces is investigated. As temperature ranges from $T_{h}$ to $T_{c}$ in the experimental set-up and $T_{h}<310 \mathrm{~K}$, surface radiation involved in it is low temperature radiation in infra-red. Due to the fact that dry air is the working fluid, fluid medium is considered as transparent for low temperature radiation. Because polycarbonate forming the front and rear walls is opaque for low temperature radiation, the cavity surfaces are supposed to be grey, diffuse and opaque, i.e. surface emissivities, $\epsilon_{i}$, and their absorptivity, $\alpha_{i}$, are independent of wavelengths and directions, furthermore $\epsilon_{i}=\alpha_{i}$. Emissivities of the cavity internal surfaces have been measured: for the polished aluminium surfaces (the vertical active walls) $\epsilon=\alpha=0.09$, for the aluminium films covering polyurethane foam $\epsilon=\alpha=$ 0.18 and for the polycarbonate surfaces $\epsilon=\alpha=0.97$. Note that the aluminium films covering polyurethane foam are separated from active walls and heat conduction in them are neglected in numerical simulations: they are supposed to only take part in surface radiation.

\section{Mathematical formulation}

The physical problem defined above involves natural convection in air, heat conduction in the horizontal insulating walls made of polyurethane foam and surface radiation between the cavity internal surfaces. In the following a brief mathematical description in the scope of DNS approach is given. Concerning the LES approach, details can be found in [23, 22] and only boundary conditions are specified.

\subsection{Boussinesq equations in air}

As $\Delta T=15 \mathrm{~K}$, we can suppose air flows in cavity are governed by the unsteady NavierStokes equations under Boussinesq approximation. Using the temperature difference $\Delta T=T_{h}-T_{c}$ and the mean temperature $T_{0}$, we define reduced temperature $\Theta=$ 
$\left(T-T_{0}\right) / \Delta T$. Using the cavity height as reference length, thermal diffusivity of air and Rayleigh number $R a$, we define reference velocity as $\kappa R a^{1 / 2} / H$. The unsteady NavierStokes equations governing air flows in the cavity read in dimensionless form:

$$
\begin{aligned}
0 & =\frac{\partial u}{\partial x}+\frac{\partial v}{\partial y}+\frac{\partial w}{\partial z} \\
\frac{\partial u}{\partial t}+u \frac{\partial u}{\partial x}+v \frac{\partial u}{\partial y}+w \frac{\partial u}{\partial z} & =-\frac{\partial p}{\partial x}+\frac{\operatorname{Pr}}{R a^{1 / 2}}\left(\frac{\partial^{2}}{\partial x^{2}}+\frac{\partial^{2}}{\partial y^{2}}+\frac{\partial^{2}}{\partial z^{2}}\right) u \\
\frac{\partial v}{\partial t}+u \frac{\partial v}{\partial x}+v \frac{\partial v}{\partial y}+w \frac{\partial v}{\partial z} & =-\frac{\partial p}{\partial y}+\frac{\operatorname{Pr}}{R a^{1 / 2}}\left(\frac{\partial^{2}}{\partial x^{2}}+\frac{\partial^{2}}{\partial y^{2}}+\frac{\partial^{2}}{\partial z^{2}}\right) v \\
\frac{\partial w}{\partial t}+u \frac{\partial w}{\partial x}+v \frac{\partial w}{\partial y}+w \frac{\partial w}{\partial z} & =-\frac{\partial p}{\partial z}+\frac{\operatorname{Pr}}{R a^{1 / 2}}\left(\frac{\partial^{2}}{\partial x^{2}}+\frac{\partial^{2}}{\partial y^{2}}+\frac{\partial^{2}}{\partial z^{2}}\right) w+\operatorname{Pr} \Theta \\
\frac{\partial \Theta}{\partial t}+u \frac{\partial \Theta}{\partial x}+v \frac{\partial \Theta}{\partial y}+w \frac{\partial \Theta}{\partial z} & =\frac{1}{R a^{1 / 2}}\left(\frac{\partial^{2}}{\partial x^{2}}+\frac{\partial^{2}}{\partial y^{2}}+\frac{\partial^{2}}{\partial z^{2}}\right) \Theta
\end{aligned}
$$

where $u, v$ and $w$ are the velocity components in respectively $x, y$ and $z$ directions. The above equations are defined in $(x, y, z) \in\left[0, A_{x}\right] \times\left[0, A_{y}\right] \times[0,1]$.

\subsection{Heat conduction in polyurethane foam}

Dimensionless equation of heat conduction in polyurethane foam reads:

$$
\frac{\partial \Theta}{\partial t}=\frac{\kappa_{f}}{\kappa} \frac{1}{R a^{1 / 2}}\left(\frac{\partial^{2}}{\partial x^{2}}+\frac{\partial^{2}}{\partial y^{2}}+\frac{\partial^{2}}{\partial z^{2}}\right) \Theta
$$

where $\kappa_{f}$ is thermal diffusivity of polyurethane foam. It is defined in $(x, y, z) \in\left[0, A_{x}\right] \times$ $\left[0, A_{y}\right] \times\left[-A_{f}, 0\right]$ (the bottom insulating wall) and $\left[0, A_{x}\right] \times\left[0, A_{y}\right] \times\left[1,1+A_{f}\right]$ (the top insulating wall).

\subsection{Low temperature surface radiation}

Given a temperature distribution on the cavity internal surfaces and the above assumptions, the surface radiation problem in a differentially heated cavity is fully described by the following linear system governing the radiosity, $\tilde{J}_{i}\left(\mathrm{~W} / \mathrm{m}^{2}\right)$ :

$$
\tilde{J}_{i}=\left(1-\epsilon_{i}\right) \sum_{j=1}^{m} F_{i j} \tilde{J}_{j}+\epsilon_{i} \sigma T_{i}^{4} \quad(i=1,2, \cdots, m)
$$

where $\sigma$ is Stefan-Boltzmann constant, $m$ is the total number of isoflux and isothermal surface elements, $F_{i j}$ is geometric view factor between $i$ surface element and $j$ surface element. The net radiative heat flux resulted from surface radiation, which is defined in the normal direction of a surface element, can be calculated by:

$$
\tilde{q}_{i}=\frac{\epsilon_{i}}{1-\epsilon_{i}}\left(\sigma T_{i}^{4}-\tilde{J}_{i}\right) \quad(i=1,2, \cdots, m)
$$


Equations (3) and (4) are the most elementary formulation of surface radiation [27, 20]. They are chosen for their simplicity although Equation (4) is not valid for black surfaces.

In numerical simulation of natural convection it is usual to work with dimensionless equations and heat transfer is characterized then by Nusselt number. Coupling natural convection with the surface radiation requires the same manner to make dimensionless heat flux, i.e., $q_{i}=\tilde{q}_{i} H /(\lambda \Delta T)$ and $J_{i}=\tilde{J}_{i} H /(\lambda \Delta T)$. (Note also that $(\lambda \Delta T) / H$ is the reference flux for conduction in polyurethane foam.) This leads to the following dimensionless equations:

$$
J_{i}-\left(1-\epsilon_{i}\right) \sum_{j=1}^{m} F_{i j} J_{j}=\epsilon_{i} \sigma H\left(\Theta_{i} \times \Delta T+T_{0}\right)^{4} /(\lambda \Delta T) \quad(i=1,2, \cdots, m)
$$

and

$$
q_{i}=\frac{\epsilon_{i}}{1-\epsilon_{i}}\left[\sigma H\left(\Theta_{i} \times \Delta T+T_{0}\right)^{4} /(\lambda \Delta T)-J_{i}\right] \quad(i=1,2, \cdots, m)
$$

In the literature of natural convection-surface radiation coupling, apart from the dimensionless parameters appeared in natural convection and the radiative properties of the surfaces, two more numbers are involved: $\Theta_{0}=T_{0} / \Delta T$, reference temperature ratio, and $N_{r}=H \sigma T_{0}^{4} /(\lambda \Delta T)$, radiation number, or its inverse Planck number. The experimental case investigated numerically in the present work corresponds to $\Theta_{0}=19.7$ and $N_{r}=1717.6359$. Note that when using radiation number $N_{r}$ or Planck number one has to use another dimensionless net radiative flux in order to calculate radiative Nusselt number. From the dimensionless net radiative flux used in Equations (5) and (6) radiative Nusselt number can be obtained directly by integrating $q_{i}$ or $-q_{i}$ depending on the considered walls. Note also that in experimental studies Rayleigh number is modified by changing either the cavity size or the temperature difference $\Delta T$ : any change in Rayleigh number will also modify $\Theta_{0}$ and $N_{r}$. It is therefore impossible, in experiments, to keep constant $\operatorname{Pr}, \Theta_{0}$ and $N_{r}$ simultaneously while varying only Rayleigh number. This means that, when doing numerical simulations using $\Theta_{0}$ and $N_{r}$, these numbers should be carefully chosen so that the computed cases correspond to experimentally realisable situations.

\subsection{Boundary and interface conditions}

On the cavity internal surfaces, velocity field satisfies no-slip conditions.

The thermal boundary conditions are complicated by the fact of involving surface radiation. In terms of energy conservation, adiabatic condition means the balance between convective flux and net radiative flux and on the interfaces between air and polyurethane foam net radiative flux should also be considered. The thermal conditions are then:

- $\Theta=0.5$ on the hot wall $(x=0)$ and $\Theta=-0.5$ on the cold wall $\left(x=A_{x}\right)$. 
- $\Theta=0$ at $z=-A_{f}$ and $1+A_{f}$ (on the external surfaces of the top and bottom walls).

- $-\left.\frac{\lambda_{f}}{\lambda} \frac{\partial \Theta}{\partial z}\right|_{\text {foam }}=-\left.\frac{\partial \Theta}{\partial z}\right|_{\text {air }}+q$ at $z=0$ (on the cavity bottom surface) and $\left.\frac{\lambda_{f}}{\lambda} \frac{\partial \Theta}{\partial z}\right|_{\text {foam }}=\left.\frac{\partial \Theta}{\partial z}\right|_{\text {air }}+q$ at $z=1$ (on the cavity top surface).

- Adiabatic conditions at $y=0$ and $A_{y}$ for polyurethane foam: $\frac{\partial \Theta}{\partial y}=0$ for $z<0$ and $z>1$.

- Adiabatic conditions at $y=0$ and $A_{y}$ on the surfaces of polycarbonate sheets: $-\frac{\partial \Theta}{\partial y}+q=0$ for $0<z<1$ and $y=0$ and $\frac{\partial \Theta}{\partial y}+q=0$ for $0<z<1$ and $y=A_{y}$.

where $\lambda_{f}$ is the thermal conductivity of polyurethane foam and $q$, the net radiative flux, is the solutions of Equations (6).

Note that

- for the case of conjugate natural convection (convection-conduction coupling), it suffices to set to zero the net radiative flux in the above thermal boundary conditions and spare the relevant computations of surface radiation. The usual adiabatic conditions are recovered on the front and rear walls.

- for the case of LES using the measured temperature distributions one needs only to work in air, i.e. in $(x, y, z) \in\left[0, A_{x}\right] \times\left[0, A_{y}\right] \times[0,1]$, and provide the thermal boundary conditions of Dirichlet type on the front $(y=0)$, rear $\left(y=A_{y}\right)$, top $(z=1)$ and bottom $(z=0)$ walls. The corresponding analytical expressions can be found in [24].

\subsection{Heat transfer}

In dimensionless form, the thermal conditions on the top $(z=1)$ and bottom $(z=0)$ walls represent the relationship between convective, conductive and radiative Nusselt numbers. In most of the times one is interested in the equivalent 2D-configuration Nusselt numbers in the mid-depth vertical plane at $y=A_{y} / 2$ and some experimental results are available for the comparison purpose. As indicated Parts I and II, Nusselt number averaged over cavity walls are also of interest and should also be provided for comparison.

If $\langle$. $>$ denotes time-averaging and $(\lambda \Delta T) / H$ is used as the reference flux, local time-averaged convective Nusselt number in air is defined as $\langle N u\rangle^{c}\left(x, A_{y} / 2,0\right)=$ $-\left.\frac{\partial<\Theta>}{\partial z}\left(x, A_{y} / 2,0\right)\right|_{\text {air }}$ at $y=A_{y} / 2$ and $z=0$. Local time-averaged diffusive Nusselt 
number in polyurethane foam on the same wall is defined as $\langle N u\rangle^{d}\left(x, A_{y} / 2,0\right)=$ $-\left.\frac{\lambda_{f}}{\lambda} \frac{\partial<\Theta>}{\partial z}\left(x, A_{y} / 2,0\right)\right|_{\text {foam }}$. Local time-averaged radiative Nusselt numbers is $\langle N u\rangle^{r}$ $\left(x, A_{y} / 2,0\right)=<q>\left(x, A_{y} / 2,0\right)$ at $z=0$ in the mid-depth vertical plane. The corresponding time-averaged mean Nusselt numbers, denoted respectively by ${\overline{\langle N u\rangle_{1 D}}}_{1 D}$, ${\overline{\langle N u\rangle_{1 D}}}_{1 D}$ and ${\overline{\langle N u\rangle_{1 D}}}_{1}^{r}$, are calculated by integrating local time-averaged Nusselt num\left. bers along the mid-depth line: ${\overline{\langle N u\rangle_{1 D}^{c}}}_{1 D}=\frac{1}{A_{x}} \int_{0}^{A_{x}}<N u\right\rangle^{c}\left(x, A_{y} / 2,0\right) d x$ for example. In the same way, Nusselt numbers are defined along the mid-width line on the top wall, but note that local time-averaged radiative Nusselt number is defined as $\langle N u\rangle^{r}\left(x, A_{y} / 2,1\right)=-\langle q\rangle\left(x, A_{y} / 2,1\right)$ at $z=1$ due to the fact that $q$ is in top wall's normal direction.

Let us recall that local time-averaged convective and radiative Nusselt numbers on the hot wall are defined as follows: $\left\langle N u>^{c}\left(0, A_{y} / 2, z\right)=-\frac{\partial<\Theta>}{\partial x}\left(0, A_{y} / 2, z\right)\right.$ and $<N u>^{r}\left(0, A_{y} / 2, z\right)=<q>\left(0, A_{y} / 2, z\right)$. Integrating them along the mid-depth line gives rise to the time-averaged mean Nusselt numbers, ${\overline{\langle N u\rangle_{1 D}}}_{1 D}$ and ${\overline{\langle N u\rangle_{1 D}}}_{1 D}$. Nusselt numbers are defined in the same way on the cold wall except for $\left\langle N u>^{r}\left(A_{x}, A_{y} / 2, z\right)=\right.$ $-<q>\left(A_{x}, A_{y} / 2, z\right)$.

The above Nusselt numbers are defined in the coordinate directions. A positive value means that heat flux is in the same direction as the coordinate. This explains why the definition of radiative Nusselt numbers is complicated by the fact that net radiative flux is always defined in surface's normal direction. Obviously, time-averaged mean Nusselt numbers over any active or passive surface can be obtained by integrating in the depth direction the above mean Nusselt numbers defined on lines. For example, on the hot wall ${\overline{\langle N u\rangle_{2 D}^{c}}}_{2 D}=\frac{1}{A_{y}} \int_{0}^{A_{y}} \int_{0}^{1}\langle N u\rangle^{c}(0, y, z) d z d y$. These definitions are similar to those in Parts I and II $[25,24]$ but complicated by the fact that one should distinguish conductive, convective and radiative Nusselt numbers.

\section{Numerical methods}

Equations (1), (2), (5) and (6) are coupled through boundary and interface conditions. The previous study [33] showed that the coupling between Equations (1) for convection in air and Equation (2) for conduction through interface conditions can be dealt with a domain decomposition approach. The main question concerns how to treat the interaction between Equations (5) and (6) of surface radiation and Equations (1) and (2) of convection-conduction. As flows to be investigated are turbulent, any instantaneous exact coupling requiring an iterative method would be too expensive to be feasible. A realistic idea is to decouple the equations of surface radiation from those of convectionconduction by explicit treatment of surface radiation problem in time due to the fact that time step must remain small for turbulent flows. In the following we recall briefly 
numerical methods used for solving Equations (1) and (2) and detail how to solve the surface radiation problem.

Equations (1) and (2) are discretized in time by a semi-implicit scheme: diffusion is treated implicitly and nonlinear terms are explicit. Solutions in polynomial forms are searched for these equations: spectral Chebyshev collocation methods are used. Apart from velocity-pressure coupling, Equations (1) are reduced to Helmholtz equations which are solved by total diagonalization as was suggested by Haidevogel and Zang [11]. Velocity-pressure coupling arisen in the unsteady Boussinesq equations is handled by projection method. Details of solving 3D unsteady Boussinesq equations can be found in [34].

In $z$-direction the layers of polyurethane foam are considered as sub-domains and Equations (2) are discretized in space by using a different grid. Together with interface conditions, the discretized energy equations are a mono-dimensional domain decomposition problem which can be solved either by a direct method or matrix influence method (see [33] for details) provided that $q$, net radiative heat flux, appeared in the boundary and interface conditions is known. Explicit treatment of $q$ leads to the following conditions at time step $n+1$ :

$$
\begin{aligned}
& -\left.\frac{\lambda_{f}}{\lambda} \frac{\partial \Theta^{n+1}}{\partial z}\right|_{\text {foam }}=-\left.\frac{\partial \Theta^{n+1}}{\partial z}\right|_{\text {air }}+\left(2 q^{n}-q^{n-1}\right) \text { at } z=0 \text { and }\left.\frac{\lambda_{f}}{\lambda} \frac{\partial \Theta^{n+1}}{\partial z}\right|_{\text {foam }}= \\
& \left.\frac{\partial \Theta^{n+1}}{\partial z}\right|_{\text {air }}+\left(2 q^{n}-q^{n-1}\right) \text { at } z=1 .
\end{aligned}
$$

- Adiabatic conditions at $y=0$ and $A_{y}: \frac{\partial \Theta^{n+1}}{\partial y}=0$ for $z<0$ and $z>1,-\frac{\partial \Theta^{n+1}}{\partial y}+$ $\left(2 q^{n}-q^{n-1}\right)=0$ for $0<z<1$ and $y=0$ and $\frac{\partial \Theta^{n+1}}{\partial y}+\left(2 q^{n}-q^{n-1}\right)=0$ for $0<z<1$ and $y=A_{y}$.

which allow to get temperature field at time step $n+1$, the rhs of Equations $(5), J_{i}^{n+1}$ and $q_{i}^{n+1}$.

Due to the fact that the cavity internal surfaces are not isothermal except for the two vertical active walls, we have to, in order to set up the surface radiation problem (3), discretize the non-isothermal cavity internal surfaces. It means that $m$, the number of surfaces elements, is no longer equal to the number of cavity surfaces and must be much larger than it.

Given a grid number of $N I \times N J \times N K$ for solving the Navier-Stokes equations (1), one gets $2(N I \times N J+N I \times N K+N J \times N K)$ surface elements. If $N I=N J=N K=100$ for example, the number of surfaces elements, which is also the dimension of the discrete radiation problem, is equal to $6 \times 10^{4}$ (very huge indeed!). Although a grid number of $10^{6}$ is common for DNS and LES, it is clear that the discrete radiation problem resulting from the same Navier-Stokes mesh still has a very huge dimension. It is therefore impossible 
to couple DNS or LES with surface radiation problem by using the same surface mesh as the one used for solving the Navier-Stokes equations.

It seemed to us that one way to deal with the convection-surface radiation coupling is to use a coarser surface mesh for the discrete radiation problem. As a differentially heated cavity possesses two isothermal vertical walls, we used a strong assumption that energy incident on and leaving these surfaces is uniform and they can thus be considered as two surface elements for the radiative problem. Although this helps considerably to reduce the dimension of surface radiation problem, it prevents us from investigating the local radiative exchange on the two vertical active walls. In this way if we take every other point of the Navier-Stokes grid on the cavity internal surfaces, with $N I=N J=$ $N K=100$ for example, the dimension of discrete surface radiation problem, equal to $2(1+N I \times N J / 4+N I \times N K / 4)=10002$, becomes more reasonable for using a direct solver. In practice for $R a=1.5 \times 10^{9}$ a surface mesh of $2(1+N I \times N J / 8+N I \times N K / 16)$ has been used for the surface radiation problem.

Obviously a coarser surface mesh for surface radiation problem imposes a polynomial interpolation of the net radiative flux from the radiation surface mesh onto the Navier-Stokes collocation mesh. As the mesh for surface radiation problem is also Gauss points, the corresponding interpolation is a uniform approximation and can be used. Note, however, that net radiative flux is an averaged quantity over each surface element, the most consistent approach requires computations of averaged temperature on each radiation surface element and deconvolution of net radiative flux from the radiation surface mesh onto the Navier-Stokes collocation mesh. In the present study we considered the inner-most pointwise temperature on a radiation surface element to be the averaged temperature over this element and the net radiative exchange over this element to be the pointwise heat flux at the inner-most point. Further investigation should be carried out in the future in order to improve the present approach.

Equations (5) can be put into matrix form $A J=b$ where $A_{i j}=\delta_{i j}-\left(1-\epsilon_{i}\right) F_{i j}$ and $b_{i}=$ $\epsilon_{i} \sigma H\left(\Theta_{i} \times \Delta T+T_{0}\right)^{4} /(\lambda \Delta T)$. Solving the surface radiation problem consists of calculating $F_{i j}$ and finding solutions of the linear system $(5) . F_{i j}$ is calculated by combining 5 point Gauss-Legendre quadrature with analytical integration : easy integrations are done analytically and the remaining is completed by Gauss-Legendre quadrature. The quality of $F_{i j}$ calculation is checked by $\sum_{j=1}^{m} F_{i j}-1<10^{-4}$. Note that matrix $A$ is diagonally dominant for $\epsilon_{i}>0$ and that iterative methods such as Jacobi, Gauss-Seidel and GMRes can be used to solve Equations (5), nevertheless in the present study $A$ is inverted and $J$ is obtained directly by doing matrix-vector product, $A^{-1} b . A^{-1}$ is obtained by using Lapack routines and $A^{-1} A=I$ is checked on round-off level. 


\section{$5 \quad$ Results and discussions}

\subsection{Experimental uncertainties}

As numerical results will be compared with experimental data [21], it is important to provide measurement uncertainties. Temperature is measured by calibrated K-type micro-thermocouples of $25 \mu \mathrm{m}$ and velocity measurements are obtained with a $5 \mathrm{~W}$ twocomponent Argon Laser Doppler Anemometer in backscatter mode. Most of temperature and velocity profiles are measured in the mid-depth plane of the central cavity at various vertical positions. Both temperature and velocity measurements made use of a Charlyrobot transverse system with a position uncertainty of $10 \mu \mathrm{m}$.

The calibrating procedure followed in [21] revealed an uncertainty of $0.12 \mathrm{~K}$ for temperature between $283.15 \mathrm{~K}$ and $323.15 \mathrm{~K}$. In terms of time-averaged reduced temperature $\Theta$, the uncertainty is equal to $0.8 \%$. The uncertainties of LDA measurements are $1 \%$ on time-averaged velocity, $5 \%$ on standard deviation and $0.1 \mathrm{~mm}$ on the measured position. More details can be found in [21].

\subsection{Numerical parameters}

For solving the Navier-Stokes equations (1) and heat conduction equation (2) in the horizontal walls, the following parameters are used: $N I=120, N J=90, N K=180$ and $N K_{f}=20 .(N I+1) \times(N J+1) \times(N K+1)=1,992,991$ is the number of grid points in the working fluid-air. As $N K_{f}$ is applied to the insulating material-polyurethane foam, the corresponding grid points for temperature field in both fluid and insulating foam are then equal to $(N I+1) \times(N J+1) \times\left(N K+2 N K_{f}+1\right)=2,433,431$. Using this grid, spectral coefficients of Chebyshev polynomials have been checked: the level of the highest frequencies is below $10^{-5}$ for instantaneous fields and $10^{-6}$ for time-averaged ones.

The surface mesh for the radiative problem is $N I / 4, N J / 2$ and $N K / 4$ respectively in $x-, y-$ and $z-$ directions (except for the hot and cold walls which are only two surface elements due to the fact that they are isothermal). The dimension of surface radiation problem is equal to $2(1+N I \times N J / 8+N I \times N K / 16)=5402$. (It means that $A\left(A^{-1}\right)$ has 29,181,604 elements and takes 233.45 Megabytes in computer memory.) Due to the fact that for a given problem with fixed parameters $A^{-1}$ needs to be computed only once, solving the surface radiation problem does not increase much computation cost: numerical simulations have been performed on a NEC SX5 vector computer by using only one processor. The corresponding performance of 7.1 GFlops in average means a speed of 5.25 seconds of mono-processor CPU time per time step. As the dimensionless time step used is equal to $1.5 \times 10^{-3}$, simulations of one dimensionless time unit take approximately one hour of mono-processor CPU time. Note also that, although the 
number of surface elements used is relatively limited, it is not possible to use more surface elements and discuss mesh sensitivity as a finer surface mesh with $N I / 2, N J$ and $N K / 2$ will use 21604 surface elements and exceed completely the capacity of one single processor (a 3.7 Gigabytes matrix to be inverted). This is also the reason why each isothermal wall has been considered as a single surface element.

\subsection{Simulations performed}

The very first numerical simulation we performed was a LES using the measured temperature distributions on the four passive walls. The aim was to show that numerical prediction should be reasonable provided that the thermal boundary conditions of Dirichlet type are realistic. As the LES yielded numerical results in agreement with the experimental data [24], several DNS were performed in order to understand from where these realistic boundary conditions come.

Using numerical results obtained at $R a=1.5 \times 10^{9}$ for the idealized cavity with adiabatic passive walls, the first DNS has been done for the case of conjugate natural convection (convection-conduction coupling). Although the convection-conduction coupling improves considerably numerical prediction of temperature distribution on the top and bottom walls, the discrepancy observed on the thermal stratification remains unchanged. Three DNS have been then set up for the coupling of natural convection with conduction in the insulating walls and surface radiation at the same Rayleigh number of $1.5 \times 10^{9}$. The first one has been performed with guessed emissivities of $\epsilon=0.3$ for the active walls and the top and bottom surfaces (thin aluminium film). This simulation has been done over 378 units of dimensionless time and two sets of turbulent statistics over 108 time units (one from 162 to 270 and another from 270 to 378 ) have been done in order to check their dependence on integration time: the fact that very small difference between the two sets of data has been observed leads us to the conclusion that the time asymptotic flow regime was reached. The second one has been performed for more than 200 time units by using the $\epsilon=0.2$ in order to check the influence of the emissivities on numerical results. After the emissivities of the active walls and the top and bottom surfaces have been measured, the last one has been performed using $\epsilon=0.09$ for the active walls and $\epsilon=0.18$ for the top and bottom surfaces for more than 300 time units. Time-averaged fields and turbulent statistics have been obtained for the last 150 time units. Table 1 summarises the numerical simulations performed.

\subsection{Convection-conduction coupling}

The DNS performed for convection-conduction coupling (S2) revealed that heat conduction in the insulating walls does influence positively the temperature distributions on the top and bottom wall as indicated by Figure 2: significant improvement on temperature 
distribution is observed in comparison with adiabatic horizontal walls. Nevertheless, numerical predictions still differ very much from the experimental data. With respect to the thermal stratification, there is no improvement: temperature profile near the cavity center remains unchanged. But it is important to note that heat conduction in the insulating walls cools down fluid near the top wall and heats it up near the bottom wall.

Despite the important effects of heat conduction in the horizontal walls on the numerical results, which are in agreement with the work of Omri and Galanis [18], the discrepancy observed on temperature distribution and thermal stratification still remains. This leads us to the conclusion that heat conduction is not the only physical phenomenon responsible for the discrepancy. Heat conduction in the insulating walls is important and should be considered by numerical studies (this is in agreement with the observation of Omri and Galanis [18]), however it is not important enough to change flow structures in the cavity core. Therefore the DNS of convection-conduction coupling was not pursued and no other results are presented here.

\subsection{Convection-conduction-radiation coupling}

As the coupling of convection with conduction in the horizontal walls did not improve numerical prediction of the thermal stratification in the cavity core, surface radiation was included in the coupling process using DNS approach. Three simulations (S3, S4 and S5) have been performed and they differ only in the wall emissivities. Only S5 has been conducted with the measured emissivities of the active walls $(\epsilon=0.09)$ and the aluminium films $(\epsilon=0.18)$. Despite of the different values of the wall emissivities, the results of the simulations S3 and S4 differ only slightly from those of simulation S5 in terms of the thermal stratification and time-averaged profiles. Some results of S4 are displayed in Figures 3 and 4 in order to show it. In the remaining of the paper, only the results of S5 will be presented and discussed in detail.

Figure 3 displays temperature distributions in the mid-depth vertical plane on the internal horizontal walls and at the mid-width $\left(x=A_{x} / 2\right)$. It shows that numerical results are strongly improved by adding surface radiation to the convection-conduction coupling because numerical prediction is almost in perfect agreement with the experimental measurements. The LES using measured temperature distributions on the passive walls also predicts well the thermal stratification in the cavity core despite the larger peak values of temperature in the horizontal boundary layers. In terms of the thermal stratification $\left(\frac{\partial<\Theta>}{\partial z}\right)$ in the cavity core, the experimental result is equal to 0.37 , the DNS (S5) and LES (S1) provided respectively 0.33 and 0.42 while DNS (S4) yielded a value in between. The agreement is really reasonable and satisfying compared with $O(1)$ of the previous studies $[21,22,23]$. 


\subsubsection{Heat transfer}

In order to explain how surface radiation reduces the thermal stratification in the cavity, heat transfer is first presented. In traditional 2D studies, heat transfer is presented in terms of local and mean Nusselt numbers. In 3D cases, the equivalent 2D configuration is the mid-depth vertical plane and in most of $3 \mathrm{D}$ investigations particular attention is paid to the equivalent Nusselt numbers.

Figure 4 depicts the numerical and experimental local Nusselt numbers averaged in time on the hot wall in the mid-depth vertical plane and shows a good agreement between them. This agreement is also supported by the values of time-averaged mean Nusselt numbers listed in Table 2. (Time-averaged global Nusselt numbers averaged over wall surfaces are listed in Table 3.) Time-averaged local Nusselt numbers along the top wall is displayed in Figure 5: reasonable agreement between numerical prediction and experimental measurement is observed for the convective Nusselt number (see also Table 2 for mean values). As far as the horizontal walls are concerned, Figure 5 and Table 2 show that ${\overline{\langle N u\rangle_{1 D}}}_{1 D}$ and ${\overline{\langle N u\rangle_{1 D}}}_{1 D}^{d}$ are positive, while ${\overline{\langle N u\rangle_{1 D}}}_{1}$ is negative. Near the top wall, fluid is cooled down not only by heat conduction in the insulating wall but also by surface radiation; near the bottom wall, fluid is heated not only by heat conduction but also by surface radiation. Figure 6 showing net radiative flux on the horizontal walls confirms the above observation: net radiative flux is essentially positive on the top wall and leaves it; net radiative flux is essentially negative on the bottom wall and arrives at it. This means that surface radiation plays the same role as heat conduction in the insulating walls, that is pumping energy from hot fluid in the upper part of the cavity and supplying energy to cold fluid in the lower part of the cavity. Therefore, both heat conduction and surface radiation tend to decrease the thermal stratification in the cavity through the horizontal walls.

Time-averaged net radiative flux on the front wall is shown in Figure 7. A similar distribution is observed on the rear wall due to the temperature symmetry. Net radiative flux is approximately positive on the top part of the front wall and the maximum value is located in the top hot corner. It is negative on the bottom part of the front wall and the minimum value is located in the bottom cold corner. This means that both the front and rear walls lose energy through surface radiation on the top parts and receive energy through surface radiation on the bottom parts. Surface radiation drains energy from hot fluid through the top parts of the front and rear walls and supplies it to cold fluid through the bottom parts of the front and rear walls. This also tends to decrease the thermal stratification in the cavity core.

In summary, it is clear that heat conduction in the insulating walls and surface radiation are both responsible for the weak thermal stratification observed in the cavity core. The effects of heat conduction are only limited in the top and bottom parts of the cavity, but the effects of surface radiation are more global as they are achieved through 
the four passive walls.

\subsubsection{Time-averaged flow structure (profiles)}

Vertical distribution of $\langle u\rangle$ velocity at mid-width and mid-depth of the cavity is shown in Figure 8. Experimental measurement indicates a weak boundary layer flow along the horizontal walls and a return flow outside the boundary layers. Near the bottom wall, for example, the boundary layer thickness is equal to about 0.1 and the minimum velocity is equal to about -0.06 , the return flow extends from $z=0.1$ to about $z=0.3$ and the maximum velocity is equal to 0.03 . Let us recall that in previous studies

- Numerical simulations performed with adiabatic horizontal walls yielded results which did not agree with experimental measurement not only in the horizontal boundary layer but also in the return flow region [22, 25].

- Numerical simulations performed with measured temperature distributions on the horizontal walls yielded results which agreed better with experimental measurement in the horizontal boundary layer, but discrepancy is still important in the return flow region $[22,25,24]$.

- The LES using the measured temperature distributions on the 4 passive walls (the FRC configuration) predicts a stronger horizontal boundary layer flow and a weaker return flow in a smaller region, but the agreement remains reasonable [24]

When investigating the convection-conduction-radiation coupling, numerical results of DNS reproduced well the experimental data except for the discrepancy observed on the peak velocity value in the boundary layer along the top wall. As LES makes use of measured temperature distributions on the top and bottom walls at the mid-width plane, it is interesting to know how these profiles are representative of the corresponding wall temperature. Figure 9 shows time-averaged temperature fields on the top and bottom walls and confirms that the mid-depth profiles are a good approximation for $80 \%$ of the surfaces.

Figure 10 depicts velocity and temperature profiles at $z=0.1,0.2, \cdots, 0.9$ along the vertical active walls: a global agreement is observed between numerical and experimental results especially as far as temperature profiles are concerned. Note nevertheless that at $z=0.7,0.8$ and 0.9 the DNS predicts smaller peak values of $\langle w>$ velocity than the experiment and the LES in the hot boundary layer and that the LES predicts slightly different $\langle u\rangle$ profiles at $z=0.8$ and 0.9 . Vertical flow in the hot boundary layer is reinforced by the outside horizontal flow almost everywhere except for the position $z=$ 0.2 at which the return flow occurs. Because of the return flow, important negative $<u>$ is observed at $z=0.8$ and 0.9 . It is also important to note that vertical velocity, $\langle w\rangle$, does not display any negative value outside the boundary layer and that slight negative 
vertical velocity outside the hot boundary layer characterises up to now the numerical simulations performed with adiabatic horizontal walls in $2 \mathrm{D}$ cases and adiabatic passive walls in $3 \mathrm{D}$ cases $[22]$.

The profiles in Figure 10 display well the centro-symmetry observed in pure natural convection in cavities. But rigorously there should not be any centro-symmetry because surface radiation breaks this symmetry. The only hint of the symmetry breaking comes from slight differences indicated by Figure 10 (b) on $\langle u>$ and $\langle\theta>$ outside the boundary layer.

In order to illustrate the global structure of the time-averaged flow and confirm the experimental observation [21], DNS results of velocity fields in three horizontal planes and two vertical planes are displayed in Figures 11 and 12. The three horizontal planes correspond to $z=0.05$ (near the bottom wall), 0.5 (mid-height) and 0.95 (near the top wall) and the two vertical planes to $y=0.02$ (near the front wall) and $y=0.30$ (near the rear wall). Figure 11 shows that the transverse velocity, $\langle v\rangle$, leaves the mid-depth vertical plane at $z=0.05$ and 0.95 , which agrees with the the experimental observation [21], and returns to this vertical plane at $z=0.5$, the mid-height. In the horizontal plane at $z=0.05$, the vertical velocity, $\langle w\rangle$, is positive near the front and rear vertical walls (the corresponding flow is upward) and at $z=0.95$ it is negative near these vertical walls (the corresponding flow is downward), while at the mid-height $(z=0.5)<w>$ is positive for $x<0.5$ (upward flow) and negative for $x>0.5$ (downward flow) along the vertical front and rear walls. Figure 12 indicates that vertical flow is mainly downward in the upper part and upward in the lower part near the front and rear walls. Near these vertical walls, the transverse flow (with $\langle v\rangle$ ) leaves the mid-depth vertical plane only near the top and bottom walls. Over $80 \%$ of the central part of the front and rear walls, the transverse flow which is very weak returns to the mid-depth vertical plane. This confirms also the experimental observation [21].

In time-averaged sense, the 3D flow structure can be described as follows. After turning the corner and leaving the hot vertical boundary layer, hot air flows along the top horizontal wall: most of the fluid particles arrive at the opposite wall and feed the cold boundary layer, but a small part of them flows horizontally and obliquely to the front and rear walls. These particles move then downwards along the front and rear wall and on their way downwards they return gradually towards the mid-depth vertical plane: depending on the vertical positions some of them join the return flow to the hot wall and the remaining particles move towards the cold wall. Although this $3 \mathrm{D}$ flow is weak compared with flow scales in the vertical boundary layers, it is of ultimate importance in terms of heat transfer. In fact, downward fluid particles along the front and rear walls are hot particles. When they move downwards, they transfer energy to the front and rear walls and are cooled down because the upper parts of the front and rear walls lose energy through radiation (positive net radiative flux in Figure 7) and the front and rear 
walls should drain energy from fluid particles. Similar phenomena occur in the bottom part of the cavity: cold particles move upwards along the front and rear walls and are heated by surface radiation while mixed with less cold particles. In this way, the above mentioned 3D flow mixes hot and less hot particles on the one hand and on the other hand thermal radiation through the top, bottom, front and rear walls transfers energy directly from the upper part of the cavity to the bottom part. This mechanism decreases considerably temperature difference in the vertical direction and leads to a weak thermal stratification.

\subsubsection{Turbulent statistics}

Turbulent statistics have been computed and compared with experimental measurements. Figure 13 displays the corresponding results in terms of turbulent intensity along the vertical walls in the mid-depth vertical plane.

Although time-averaged profiles show to a large extent the centro-symmetry, turbulent statistics which are less symmetrical indicate better the symmetry-breaking by surface radiation. In terms of turbulent quantities, the hot and cold boundary layers behave slightly different: the downstream of the cold boundary layer seems to be slightly more turbulent in terms of $w_{r m s}$ but slightly less turbulent in terms of $\theta_{r m s}$. The overall agreement between numerical and experimental results are good, but note that numerical simulations over-estimate turbulent intensity of temperature. Concerning the vertical velocity, $w$, turbulent intensity increases in the hot boundary layer, but for temperature turbulent quantities are first damped from $z=0.1$ to $z=0.3$ and then amplified up to $z=0.9$. More generally, the profiles of turbulent intensity take the boundary layer form of the time-averaged $w$ velocity (apart from the $w_{r m s}$ profiles at $z=0.1$ ): $\theta_{r m s}$ is strongly correlated with $\langle w\rangle$ as the peak values of $\theta_{r m s}$ locate almost at the peak positions of the time-averaged $w$ velocity, $\langle w\rangle$; peak values of $w_{r m s}$ locate outside the peak positions of $\theta_{r m s}$ and $\langle w\rangle$.

Compared with time-averaged profiles, the agreement between numerical and experimental results are less good for turbulent quantities. This can be partially explained by experimental uncertainties: inertia of the micro-thermocouples may influence the accuracy of $\theta_{\text {rms }}$ for example. It is nevertheless important to note that the current agreement is much better than the previous ones [22].

\section{Summary and concluding remarks}

For many years we have been doing comparative experimental and numerical studies in order to understand the discrepancy observed on thermal stratification in a differentially heated air-filled cavity. 
It was thought first that the discrepancy would be due to the $2 \mathrm{D}$ numerical simulations performed for 3D experimental configuration. Efforts have been made to develop 3D codes, unfortunately 3D numerical simulations did not improve the agreement between numerical and experimental results: the same discrepancy was observed on the thermal stratification [22]. Similar conclusion was also given in [29] for a cavity of aspect ratio 4. Numerical studies using temperature distributions measured on the top and bottom walls have been then proposed $[15,9,19]$. It turned out that this suggestion had only limited effect on the results in the top and bottom parts of the cavity and did not have any effect on the thermal stratification in the cavity core [22, 25]. Previous works and various reflexions led us to take into consideration heat conduction in the insulating materials and thermal radiation between the cavity internal surfaces in the present study.

Heat conduction in the insulating horizontal walls should be considered because the working fluid, air, is a good insulating material, this is also supported by a recent study [18]. The coupling of natural convection with conduction in solid materials makes use of domain decomposition techniques [33]: different materials define naturally the sub-domains and heat flux is conserved at interfaces. Numerical simulation performed by coupling natural convection with heat conduction in the insulating horizontal walls yielded results similar to those obtained by using temperature distributions measured on the internal surfaces of the horizontal walls: only results in the top and bottom parts of the cavity have been modified. The near wall distributions of $u$ velocity and temperature are considerably improved, but the discrepancy observed on the thermal stratification remains unchanged in the cavity core.

Surface radiation should be important because the front and rear walls are almost black surfaces for low temperature radiation. It modifies the meaning of adiabatic conditions and the interface conditions: adiabatic condition implies that convection flux balances net radiative flux and interface conditions on the internal surfaces of the top and bottom walls mean a balance of convection in air, conduction in the insulation and surface radiation. Surface radiation was considered by using the common radiosity formulation and assumptions that the surfaces are grey, diffusive and opaque. Net radiative flux was also treated explicitly in time in order not to use any iterative methods. The DNS has been performed for convection-conduction-radiation coupling and revealed interesting results: a good agreement between numerical prediction and experimental results is observed almost everywhere. This shows that surface radiation is an important factor that affects natural convection in air-filled cavities and that the experimental results of natural convection in air have resulted from the coupled phenomena of convection, conduction and radiation. Surface radiation reduces the thermal stratification through not only the horizontal walls but also the front and rear walls: first it cools down the top wall and heats up the bottom wall; second it drains energy from hot air 
through the top parts of the front and rear walls and supplies energy to cold air through the bottom parts of the front and rear walls. It is through the front and rear walls and the corresponding heat transfer that a downward flow and a upward flow are observed respectively along these walls near the top and bottom parts. In this sense, the 'passive' front and rear walls are far from being passive because they take part in heat transfer through thermal radiation and decrease the thermal stratification in the cavity core.

In this paper, numerical results of the LES which has been performed by using temperature distributions measured on not only the top and bottom walls but also the front and rear walls [24] are detailed and a good agreement with experimental data is shown. This means that

- On the one hand, it is not sufficient for numerical simulations to use temperature distributions measured only on the top and bottom walls in order to obtain experimentally coherent results, one should rely on experimental studies to measure also temperature distributions on the front and rear walls.

- On the other hand, it is sufficient for numerical simulations to use the measured temperature distributions on all the 'passive' walls in order not to study in detail the coupled phenomena of natural convection, heat conduction and surface radiation.

This means also that measured temperature distributions on the 'passive' walls come from the full coupling of natural convection in air, conduction in insulating material and radiation between internal surfaces.

Although the present study showed the ultimate importance of surface radiation in air-filled natural convection, it will be important to study mesh sensitivity of radiative heat transfer for the present case and revisit other well-known experimental configurations, that of Tian and Karayiannis [28] for example, in order to confirm the current observation. Given the importance of surface radiation observed in this work and the fact that surface radiation affects strongly flow structures of 2D natural convection in air-filled cavities and the corresponding onset of time-dependent flows [31], it is necessary to clarify the meaning of numerical investigations of pure natural convection flows in air as surface radiation is inherent in air natural convection flows : pursuing such studies in the future is more motivated by numerical challenge than by physical understanding. For the time being, it is clear that there need benchmark problems for natural convection-radiation coupling in 2D/3D air-filled cavities and the corresponding reference solutions.

ACKNOWLEDGMENTS : This work has been conducted under research project COCORAPHA of the French CNRS Programme Energie. Computations have been 
performed at IDRIS (Institut du Développement et des Ressources en Informatique Scientifique) under projects 0326 and 1474.

\section{References}

[1] M. Akiyama and Q.P. Chong. Numerical analysis of natural convection with surface radiation in a square enclosure. Num. Heat Trans. A, 31:419-433, 1997.

[2] C. Balaji and S.P. Venkateshan. Interaction of surface radiation with free convection in a square cavity. Int. J. Heat \& Fluid Flow, 14:260-267, 1993.

[3] C. Balaji and S.P. Venkateshan. Correlations for free convection and surface radiation in a square cavity. Int. J. Heat \& Fluid Flow, 15:249-251, 1994.

[4] M. Behnia, J.A. Reizes, and G. de Vahl Davis. Combined radiation and natural convection in a rectangular cavity with a transparent wall and containing a nonparticipating fluid. Int. J. Num. Meth. in Fluids, 10:305-325, 1990.

[5] M. Borjini, H. Ben Aissia, K. Halouani, and B. Zeghmati. Effect of radiative heat transfer on the three-dimensional buoyancy flow in cubic enclosure heated from the side. Int. J. Heat \& Fluid Flow, 23:107-118, 2008.

[6] L.C. Chang, K.T. Yang, and J.R. Lloyd. Radiation-natural convection interactions in two dimensional complex enclosures. ASME J. Heat Trans., 105:89-95, 1983.

[7] G. Colomer, M. Costa, R. Consul, and A. Oliva. Three-dimensional numerical simulation of convection and radiation in a differentially heated cavity using the discrete ordinates method. Int. J. Heat Mass Trans., 47(2):257-269, 2004.

[8] T. Fusegi and B. Farouk. Laminar and turbulent natural convection-radiation interactions in a square enclosure filled with a non-gray gas. Num. Heat Trans. A, 15:303-322, 1989.

[9] Toru Fusegi and Jae Min Hyun. Laminar and transitional natural convection in an enclosure with complex and realistic conditions. International Journal of Heat and Fluid Flow, 15(4):258 - 268, 1994.

[10] D.D. Gray and A. Giorgini. The validity of the Boussinesq approximation for liquids and gases. Int. J. Heat Mass Trans., 19:545-551, 1976.

[11] D. Haidvogel and T. Zang. The accurate solution of Poisson's equations by expansion in Chebyshev polynomials. J. Comp. Phys., 30:167-180, 1979. 
[12] C.Y. Han and Seung Wook Baek. The effects of radiation on natural convection in a rectangular enclosure divided in two partitions. Num. Heat Trans. A, 37(3):249-270, 2000.

[13] M. Kassemi and M.H.N. Naraghi. Analysis of radiation-natural convection interactions in 1-g and low-g environments using the discret exchange factor method. Int. J. Heat Mass Trans., 17:4141-4149, 1994.

[14] G. Lauriat. Combined radiation-convection in gray fluids enclosed in vertical cavities. ASME J. Heat Trans., 104:609-615, 1982.

[15] P. Le Quéré. Onset of unsteadiness, route to chaos and simulations of chaotic flows in cavities heated from the side: a review of present status. In Proceedings of the 10th IHTC Conference, pages 281-296, Washington DC, 1994. Hemisphere.

[16] S. Mergui and F. Penot. Analyse des vitesse et température de l'air en convection naturelle dans une cavité carrée différentiellement chauffée à $R a=1.69 \times 10^{9}$. Int. J. Heat Mass Trans., 40:3427-3441, 1997.

[17] A. Mezrhab and L. Bchir. Radiation-natural convection interactions in partitioned cavities. Int. J. Num. Meth. for Heat \& Fluid Flow, 8(7):781-799, 1998.

[18] M. Omri and N. Galanis. Numerical analysis of turbulent buoyant flows in enclosures: Influence of grid and boundary conditions. Int. J. Thermal Sci., 46:727-738, 2007.

[19] S.H. Peng and L. Davidson. Large eddy simulation for turbulent buoyant flow in a confined cavity. Int. J. Heat \& Fluid Flow, 22:323-331, 2001.

[20] J.F. Sacadura. Initiation aux transferts thermiques. Technique et Documentation, Paris, 1993.

[21] J. Salat. Contribution à l'étude de la convection naturelle tridimensionnelle en cavité différentiellement chauffée. PhD thesis, Univ. de Poitiers, Poitiers, France, 2004.

[22] J. Salat, S. Xin, P. Joubert, A. Sergent, F. Penot, and P. Le Quéré. Experimental and numerical investigation of turbulent natural convection in a large air-filled cavity. Int. J. Heat $\mathscr{G}$ Fluid Flow, 25(5):824-932, 2004.

[23] A. Sergent, P. Joubert, and P. Le Quéré. Development of a local subgrid diffusivity model for large eddy simulation of buoyancy driven flows: application to a square differentially heated cavity. Num. Heat Trans. A, 43:849-884, 2003. 
[24] A. Sergent, P. Joubert, S. Xin, and P. Le Quéré. Resolving the stratification discrepancy of turbulent natural convection in differentially heated air-filled cavities. Part II: end wall effects using Large-Eddy Simulation. Int. J. Heat \& Fluid Flow, doi:10.1016/j.ijheatfluidflow.2012.10.005, 2012.

[25] A. Sergent, S. Xin, P. Joubert, P. Le Quéré, J. Salat, and F. Penot. Resolving the stratification discrepancy of turbulent natural convection in differentially heated airfilled cavities. Part I: reference solutions using Chebyshev spectral methods. Int. J. Heat \& Fluid Flow, doi:10.1016/j.ijheatfluidflow.2012.08.008, 2012.

[26] A. K. Sharma, K. Velusamy, C. Balaji, and S.P. Venkateshan. Conjugate turbulent natural convection with surface radiation in air filled rectangular enclosures. Int. J. Heat Mass Trans., 50:625-639, 2007.

[27] R. Siegel and J.R. Howell. Thermal Radiation Heat Transfer. Hemisphere Publishing Co., New York, 1981.

[28] Y.S. Tian and T.G. Karayiannis. Low turbulence natural convection in an air filled square cavity, part I \& II. Int. J. Heat Mass Trans., 43:849-884, 2000.

[29] F.X. Trias, M. Soria, A. Oliva, and C.D. Perez-Segarra. Direct numerical simulation of two and three-dimensional natural convection flows in a differentially heated cavity of aspect ratio 4. J. Fluid Mech., 586:259-293, 2007.

[30] K. Velusamy, T. Sundararajan, and K.N. Seetharamu. Interaction effects between surface radiation and turbulent natural convection in square and rectangular enclosures. ASME J. Heat Trans., 123:1063-1070, 2001.

[31] H. Wang, S. Xin, and P. Le Quéré. Etude numérique du couplage de la convection naturelle avec le rayonnement de surfaces en cavité carrée remplie d'air. $C . R$. Mécanique, 334:48-57, 2006.

[32] J. Xamán, F. Hinojosa, J. Flores, and R. Cabanillas. Effect of the surface thermal radiation on turbulent natural convection in tall cavities of facade elements. Heat Mass Transfert, 45:177-185, 2008.

[33] S. Xin, M.-C. Duluc, F. Lusseyran, and P. Le Quéré. Numerical simulations of natural convection around a line-source. Int. J. Num. Meth. Heat \& Fluid Flow, 14(7):828-848, 2004.

[34] S. Xin and P. Le Quéré. An extended Chebyshev pseudo-spectral benchmark for the 8:1 differentially heated cavity. Int. J. Num. Meth. in Fluids, 40(8):981-998, 2002. 
[35] A. Yücel, A. Acharya, and M.L. Williams. Natural convection and radiation in a square enclosure. Num. Heat Trans. A, 15:261-278, 1989.

\begin{tabular}{|c|c|c|c|c|c|}
\hline Simulation & Type & $\begin{array}{c}\text { Physical } \\
\text { phenomena }\end{array}$ & $\begin{array}{c}\text { Active } \\
\text { walls }\end{array}$ & $\begin{array}{c}\text { Horizontal } \\
\text { walls }\end{array}$ & $\begin{array}{c}\text { Front and } \\
\text { rear walls }\end{array}$ \\
\hline S1 & LES & convection & $\begin{array}{c}\text { measured } \\
\text { temperature }\end{array}$ & $\begin{array}{c}\text { measured } \\
\text { temperature }\end{array}$ \\
\hline S2 & DNS & $\begin{array}{c}\text { convection } \\
\text { and conduction }\end{array}$ & & interface & adiabatic \\
\hline S3 & DNS & $\begin{array}{c}\text { convection, } \\
\text { conduction } \\
\text { and radiation }\end{array}$ & $\epsilon=0.3$ & $\begin{array}{c}\text { interface } \\
\epsilon=0.3\end{array}$ & $\begin{array}{c}\text { adiabatic } \\
\epsilon=0.97\end{array}$ \\
\hline S4 & DNS & $\begin{array}{c}\text { convection, } \\
\text { conduction } \\
\text { and radiation }\end{array}$ & $\epsilon=0.2$ & $\begin{array}{c}\text { interface } \\
\epsilon=0.2\end{array}$ & $\begin{array}{c}\text { adiabatic } \\
\epsilon=0.97\end{array}$ \\
\hline S5 & DNS & $\begin{array}{c}\text { convection, } \\
\text { conduction } \\
\text { and radiation }\end{array}$ & $\epsilon=0.09$ & $\begin{array}{c}\epsilon=0.18 \\
\text { interface } \\
\text { adiabatic } \\
\end{array}$ &
\end{tabular}

Table 1: Numerical simulations performed in the present work. S3 and S4 have been performed by using guessed emissivities of the active walls and horizontal walls.

\begin{tabular}{|c|c|c|c|c|c|c|}
\hline & \multicolumn{3}{|c|}{ Hot wall } & \multicolumn{3}{|c|}{ Cold wall } \\
\hline & EXP. & DNS & LES & EXP. & $\overline{D N S}$ & LES \\
\hline \multirow[t]{3}{*}{${\overline{<N u\rangle_{1 D}}}_{1 D}^{c}$} & 55 & 53.43 & 55.3 & 54 & 54.65 & 55.3 \\
\hline & \multicolumn{3}{|c|}{ Top wall } & \multicolumn{3}{|c|}{ Bottom wall } \\
\hline & EXP. & DNS & LES & EXP. & DNS & LES \\
\hline${\overline{\langle N u\rangle_{1 D}^{c}}}_{1}$ & 6.5 & 7.96 & 10.6 & 6.1 & 8.08 & 10.6 \\
\hline${\overline{\langle N u\rangle_{1 D}}}_{1 D}$ & & -4.85 & & & -4.97 & \\
\hline${\overline{\langle N u\rangle_{1 D}^{d}}}_{1}^{d}$ & & 3.11 & & & 3.11 & \\
\hline
\end{tabular}

Table 2: Time-averaged mean Nusselt numbers along different lines in the vertical middepth plane. On the active walls, numerical results of both DNS (S5) and LES (S1) agree well with the experimental measurements. On the internal horizontal walls, numerical simulations predict higher convective Nusselt numbers than the experiment and both numerical and experimental results have the same order of magnitude. 


\begin{tabular}{|c|c|c|c|c|}
\hline & \multicolumn{2}{|c|}{ Hot wall } & \multicolumn{2}{|c|}{ Cold wall } \\
\hline & DNS & LES & DNS & LES \\
\hline${\overline{\langle N u\rangle_{2 D}}}_{2 D}^{c}$ & 54.04 & 54.3 & 54.77 & 54.3 \\
\hline \multirow[t]{3}{*}{${\overline{<N u\rangle_{2 D}}}_{2}^{r}$} & 10.42 & & 9.81 & \\
\hline & \multicolumn{2}{|c|}{ Top wall } & \multicolumn{2}{|c|}{ Bottom wall } \\
\hline & DNS & LES & DNS & LES \\
\hline${\overline{\langle N u\rangle_{2 D}}}_{2 D}^{c}$ & 7.74 & 10.0 & 7.70 & 10.0 \\
\hline${\overline{\langle N u\rangle_{2 D}}}_{2 D}$ & -4.56 & & -4.61 & \\
\hline${\overline{<N u\rangle_{2 D}}}_{2 D}^{d}$ & 3.17 & & 3.09 & \\
\hline
\end{tabular}

Table 3: Time-averaged global Nusselt numbers over different wall surfaces. Concerning the convective Nusselt number, good agreement is observed between the DNS (S5) and LES (S1) results on the active walls. Furthermore, the DNS results showed that energy conservation is fulfilled to $0.37 \%$ in time-averaged sense as $\left({\overline{\langle N u\rangle_{2 D}}}_{2 D}+{\overline{\langle N u\rangle_{2 D}}}_{2}\right)_{\text {cold }}-$ $\left({\overline{\langle N u\rangle_{2 D}}}_{2 D}+{\overline{\langle N u\rangle_{2 D}}}^{r}\right)_{\text {hot }}+\left({\overline{\langle N u\rangle_{2 D}}}_{2}^{d}\right)_{\text {top }}-\left({\overline{\langle N u\rangle_{2 D}}}_{2 D}^{d}\right)_{\text {bottom }}$ is equal to 0.2 .

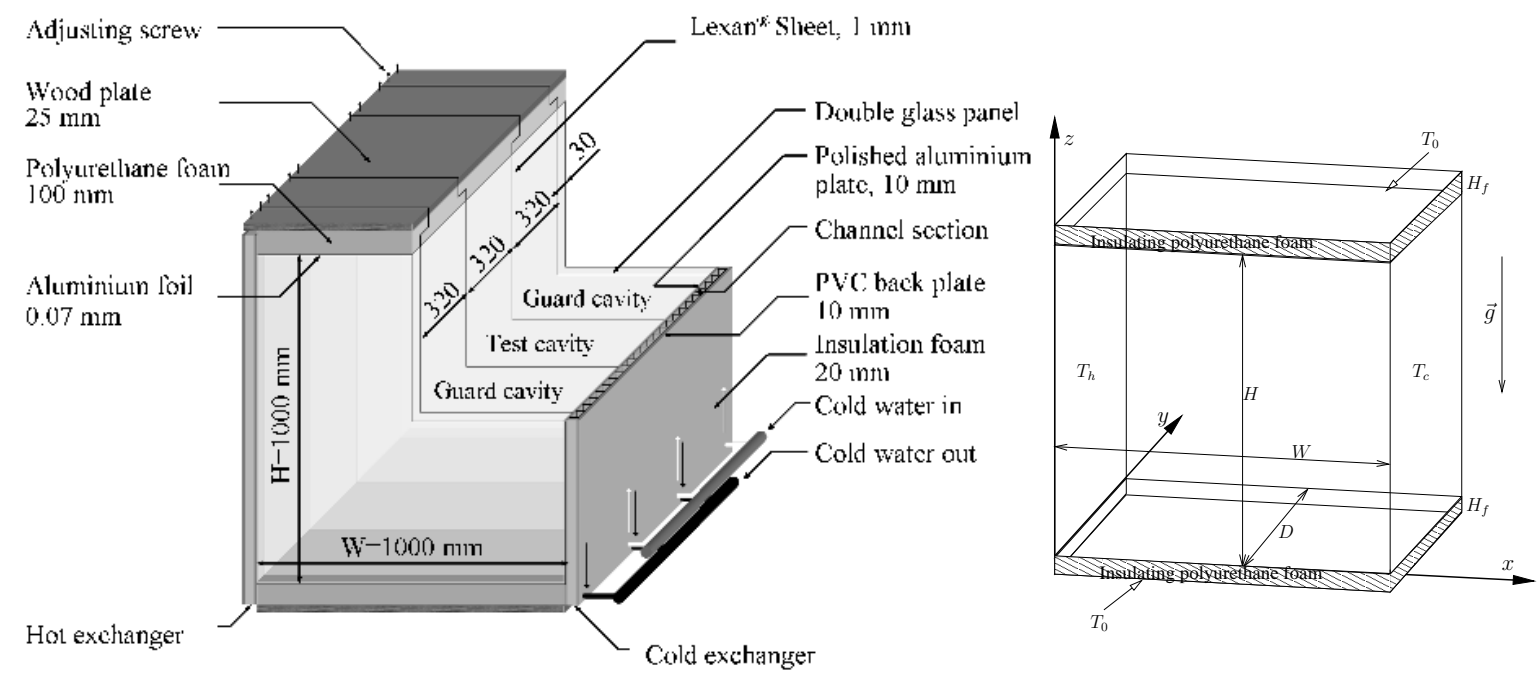

Figure 1: Experimental set-up (left) and numerically studied configuration (right). The studied configuration is an air-filled differentially heated cavity of $1 \mathrm{~m} \times 0.32 \mathrm{~m} \times 1 \mathrm{~m}$ (width $\times$ depth $\times$ height) subject to a temperature difference of $15 \mathrm{~K}\left(=T_{h}-T_{c}\right)$ and insulated on top and at bottom by polyurethane foam of $0.1 \mathrm{~m}$ thick $\left(H_{f}=0.1 \mathrm{~m}\right)$. 

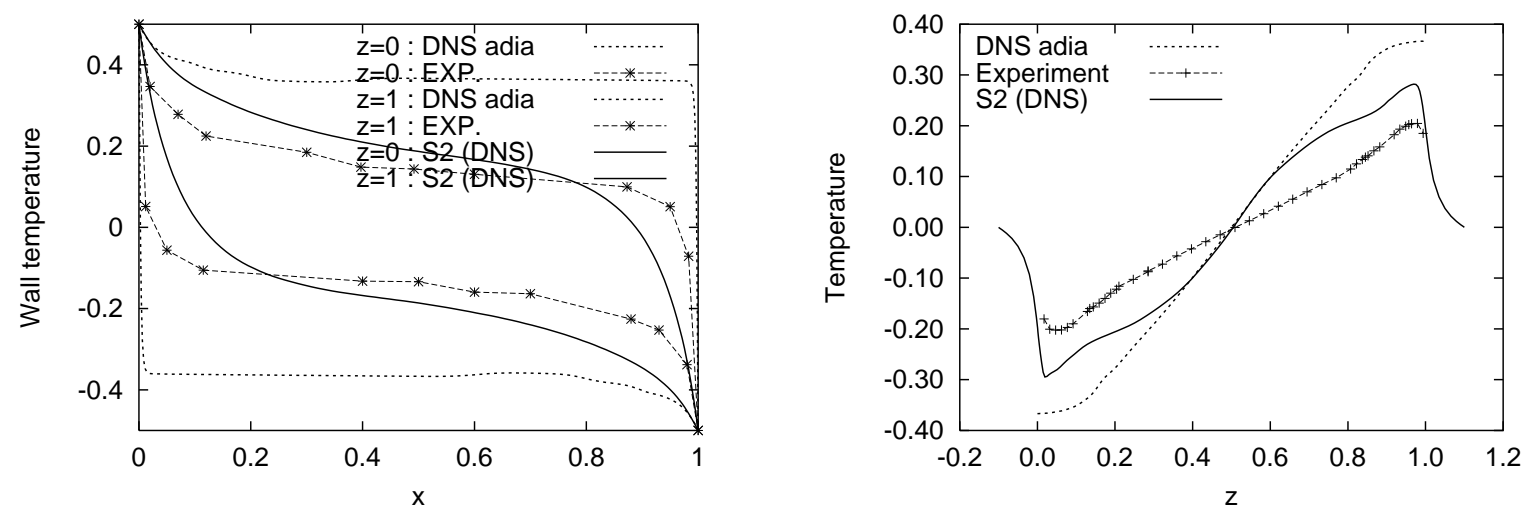

Figure 2: Time-averaged temperature profiles in the mid-depth vertical plane $\left(y=A_{y} / 2\right)$ on the internal horizontal surfaces (left) and at mid-width (right).
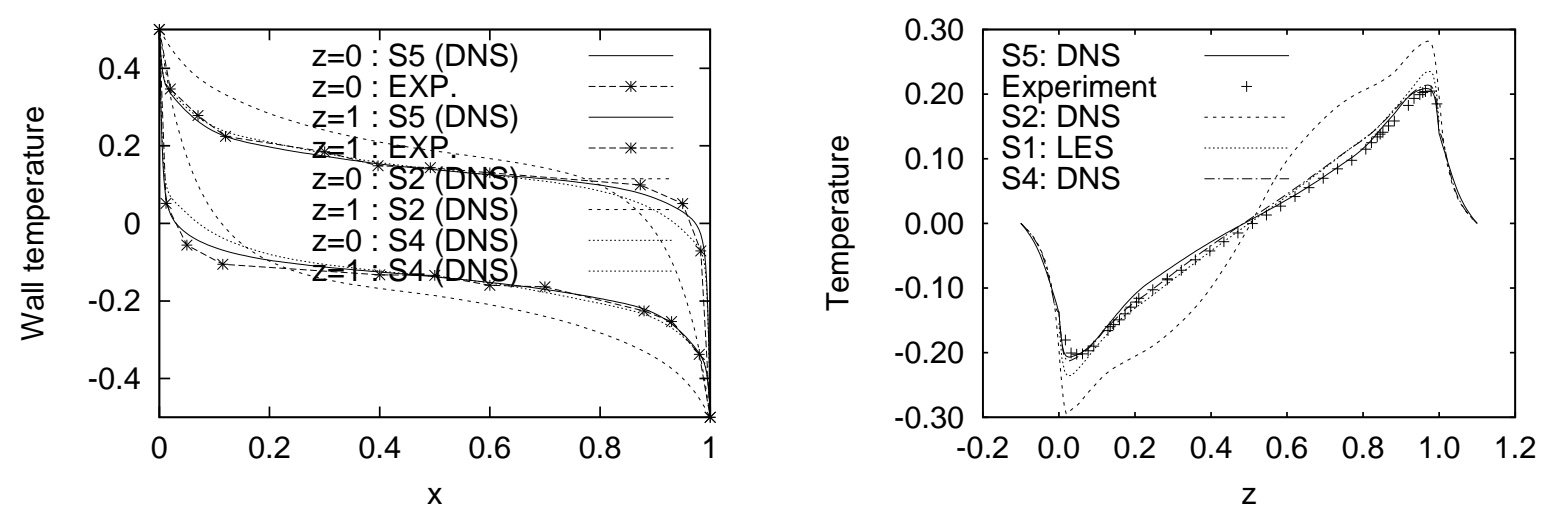

Figure 3: Time-averaged temperature profiles in the mid-depth vertical plane $\left(y=A_{y} / 2\right)$ on the internal horizontal surfaces (left) and at mid-width (right). The full convectionconduction-radiation coupling improves not only temperature distributions on the internal horizontal surfaces but also thermal stratification in the cavity core.

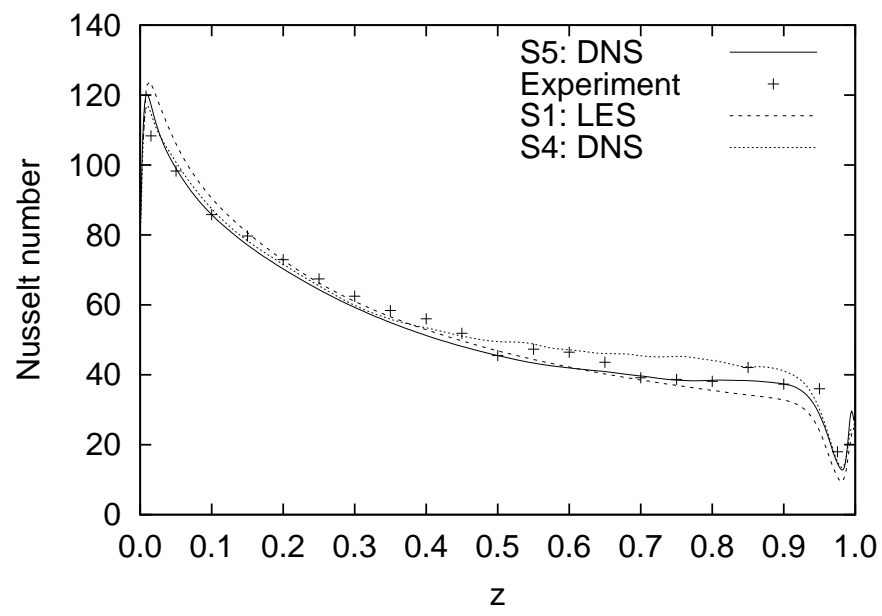

Figure 4: Time-averaged local convective Nusselt number along the hot wall $(x=0)$ in the mid-depth plane $\left(y=A_{y} / 2\right)$. A good agreement is observed between the simulations and measurements. 

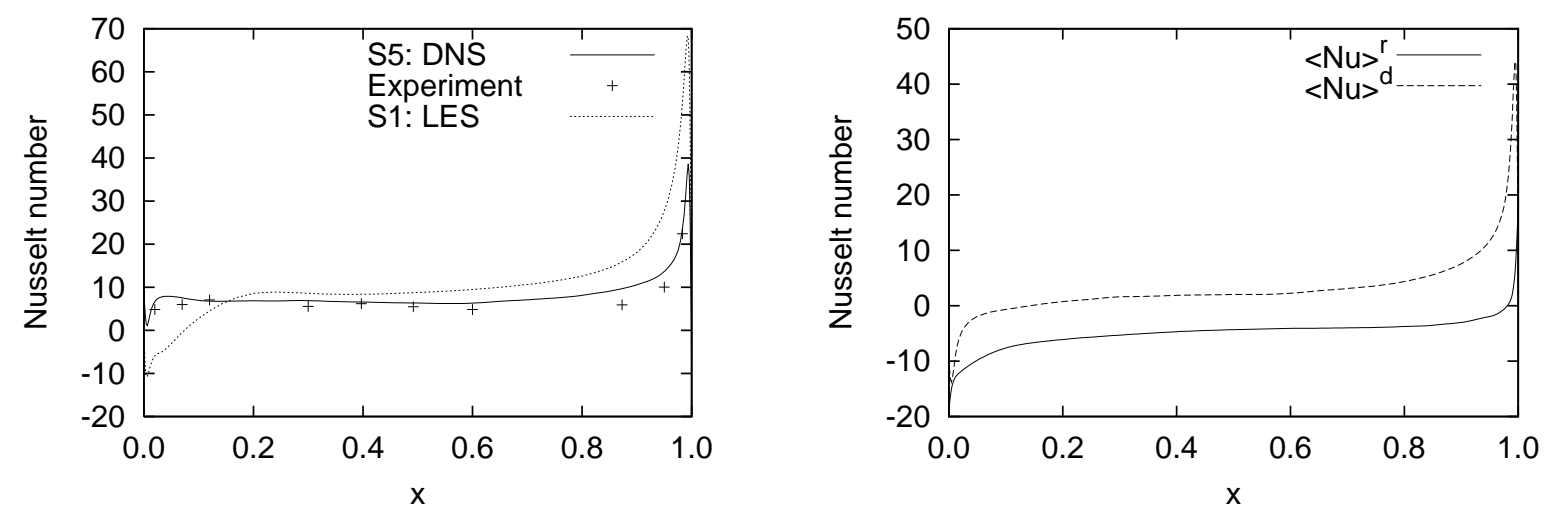

Figure 5: Time-averaged local Nusselt numbers along the top wall (convective on the left; radiative and diffusive on the right) at $z=1$ in the mid-depth vertical plane $\left(y=A_{y} / 2\right)$.
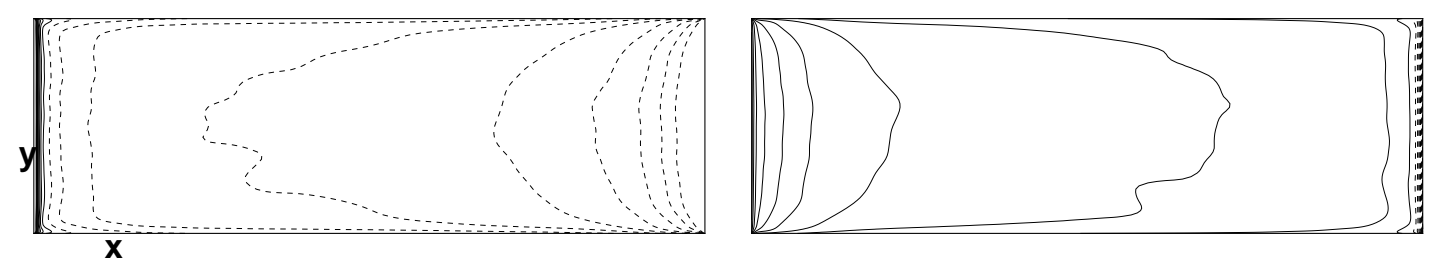

Figure 6: DNS results of time-averaged net radiative flux on the bottom (left) and top (right) walls. The continuous lines represent positive values and the dashed ones negative values. Net radiative flux is essentially negative on the bottom wall, it implies that the bottom wall receives energy through surface radiation. Net radiative flux is essentially positive on the top wall, it means that the top wall loses energy through surface radiation.

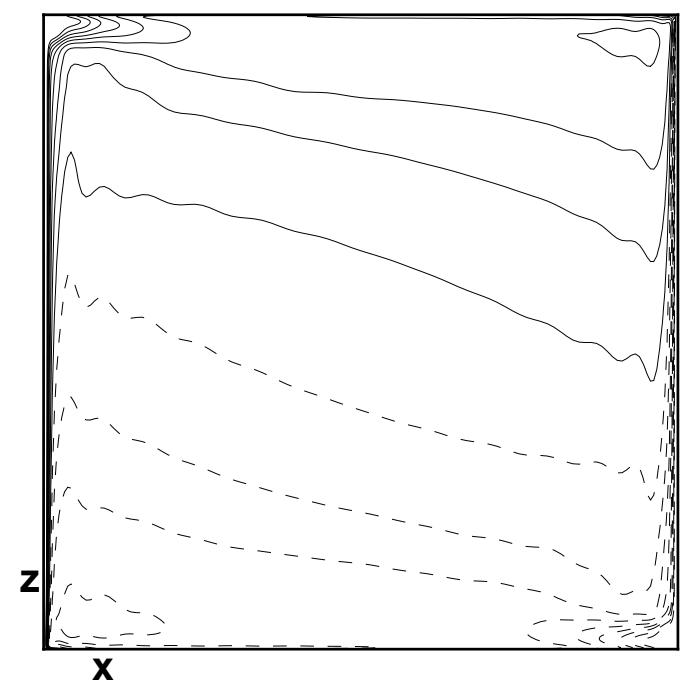

Figure 7: DNS results of time-averaged net radiative flux on the front wall $(y=0)$. Approximately, net radiative flux is positive on the top half and negative on the bottom half. This indicates that the top part of the front wall loses energy through surface radiation and drains it from hot fluid and that the bottom part receives energy through surface radiation and supplies it to cold fluid. 


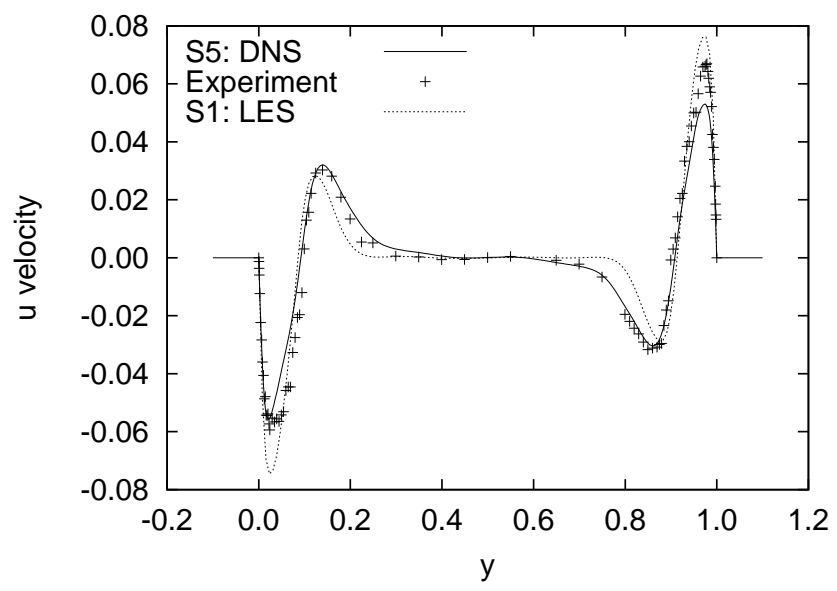

Figure 8: Time-averaged $u$ velocity, $\langle u\rangle$, at mid-width $\left(x=A_{x} / 2\right)$ and mid-depth $\left(y=A_{y} / 2\right)$.
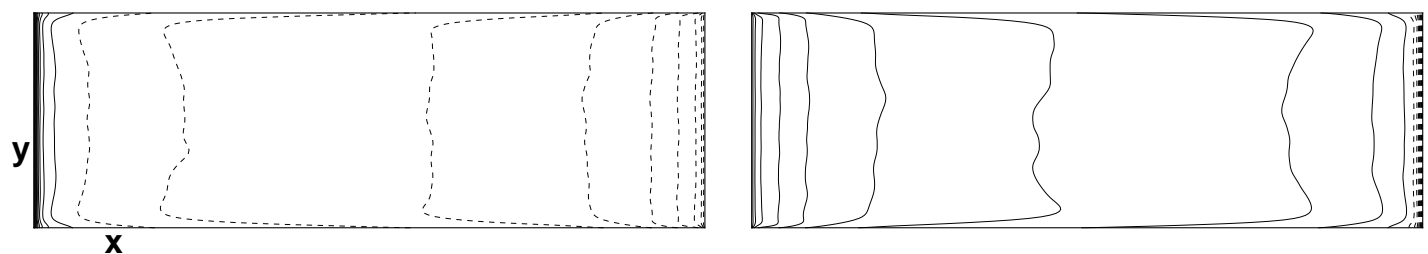

Figure 9: Time-averaged temperature on the bottom (left) and top (right) walls. 

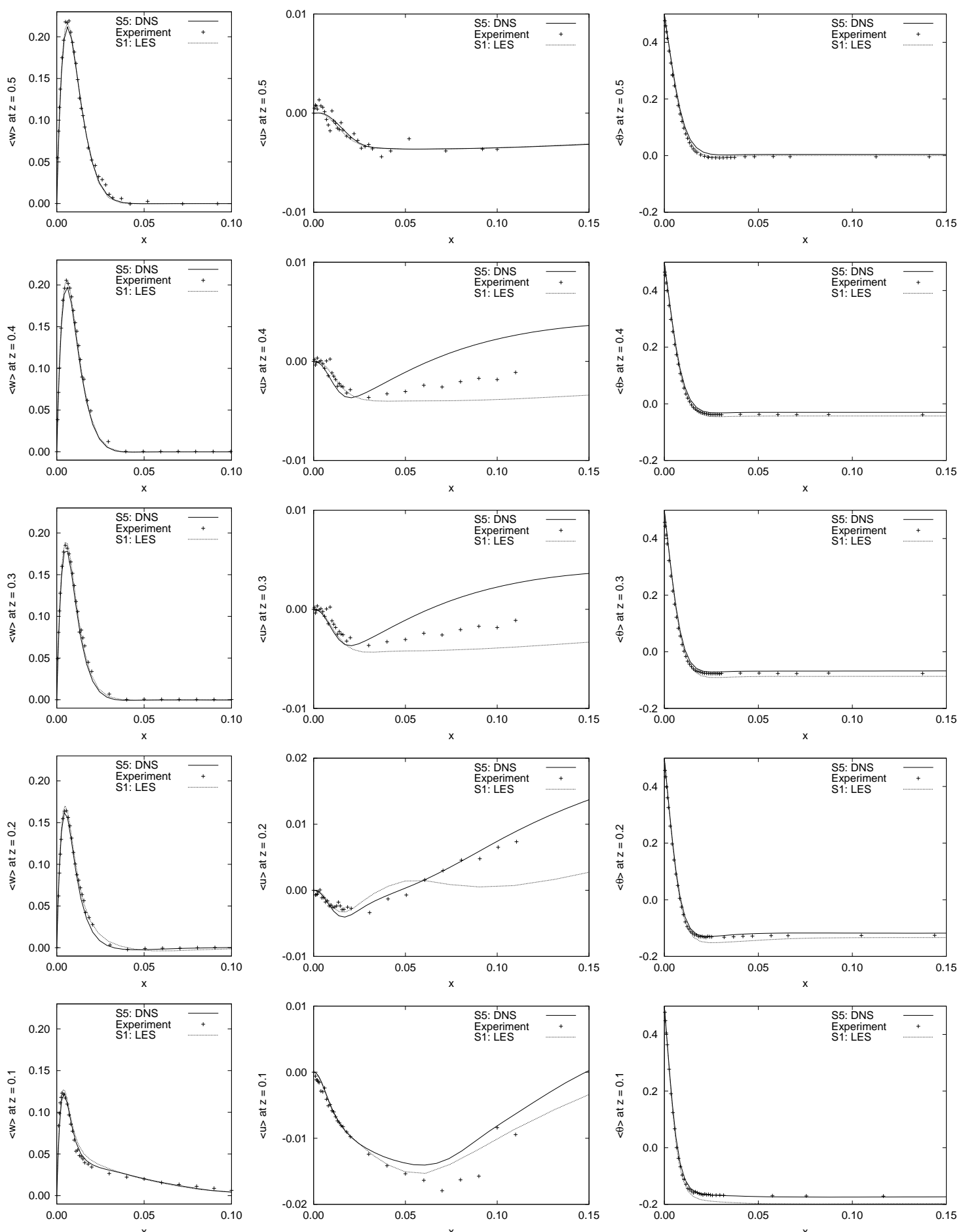

Figure 10: (a) Time-averaged profiles of velocity and temperature in the mid-depth vertical plane at $z=0.5,0.4,0.3,0.2$ and 0.1 (from top to bottom). 

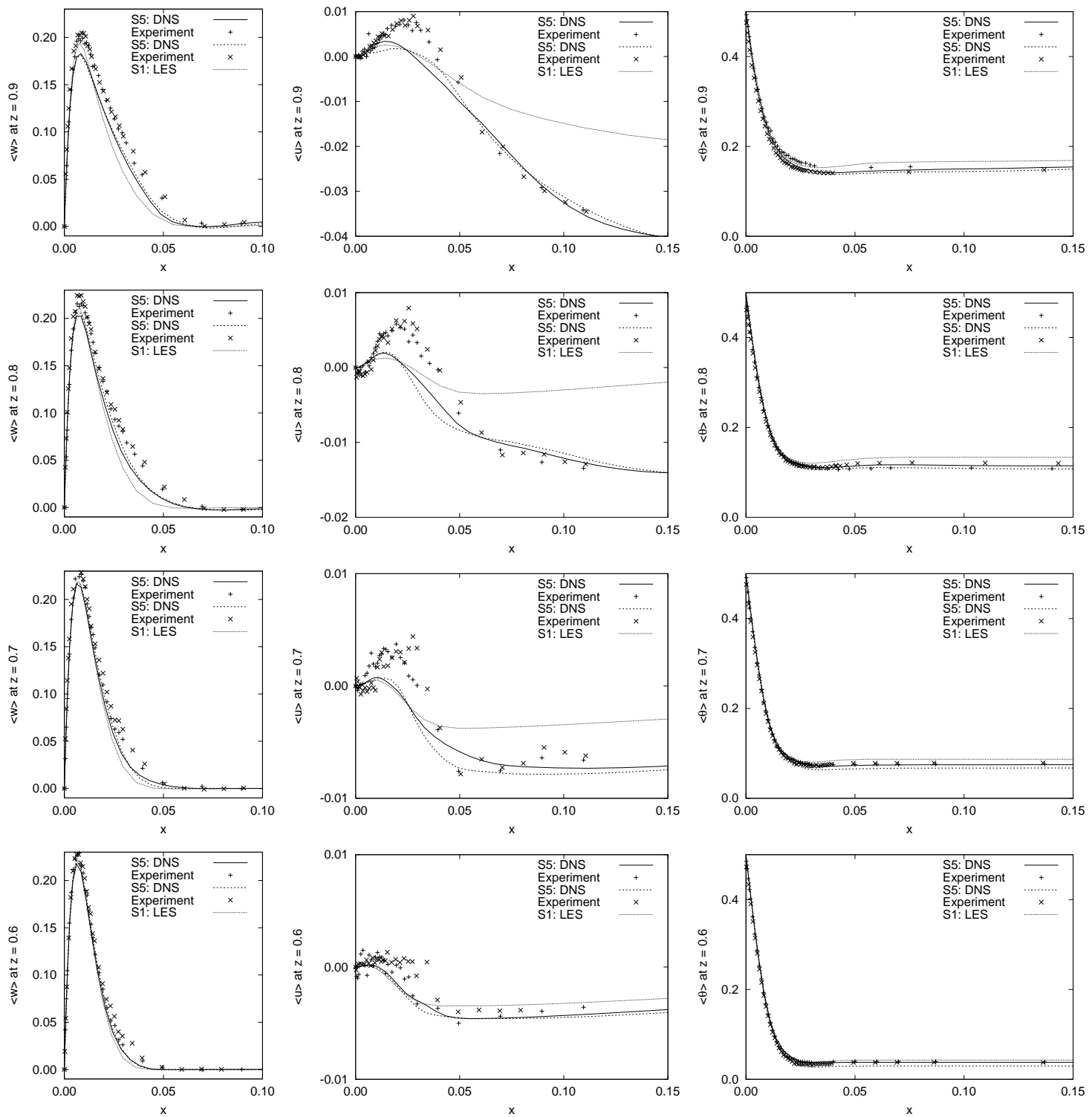

Figure 10: (b) Time-averaged profiles of velocity and temperature in the mid-depth vertical plane at $z=0.9,0.8,0.7$ and 0.6 (from top to bottom). In order to illustrate the flow centro-symmetry, profiles of velocity and temperature along the cold wall (dashed lines for the DNS results and $\times$ symbols for the experimental data) are also shown in terms of $A_{x}-x$ and $1-z$. 

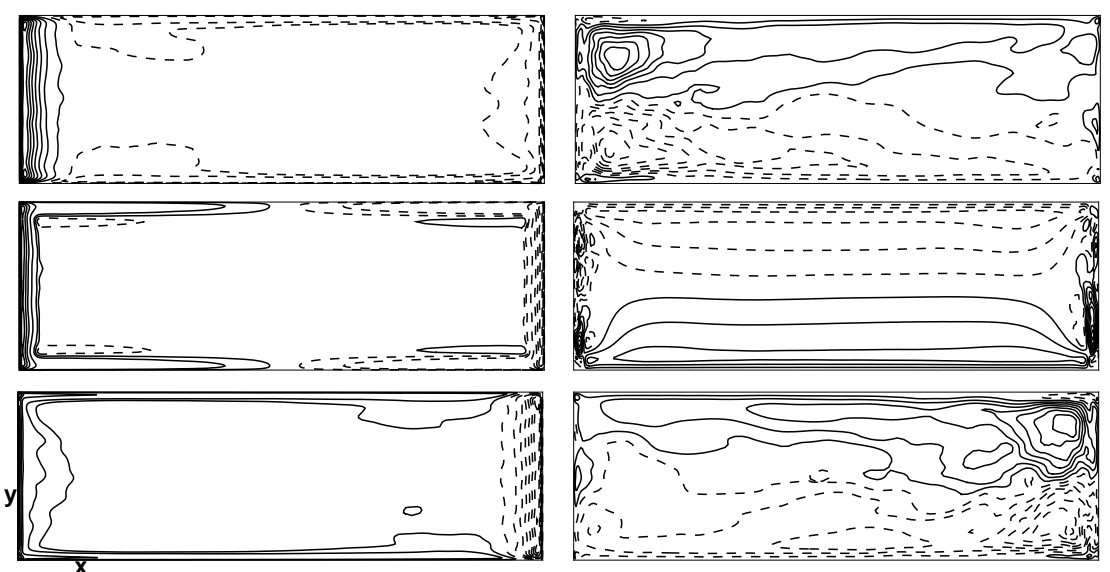

Figure 11: DNS results of $\langle w\rangle$ velocity (left) and $\langle v\rangle$ velocity (right) in the horizontal planes at $z=0.05$ (bottom), 0.5 (middle) and 0.95 (top).
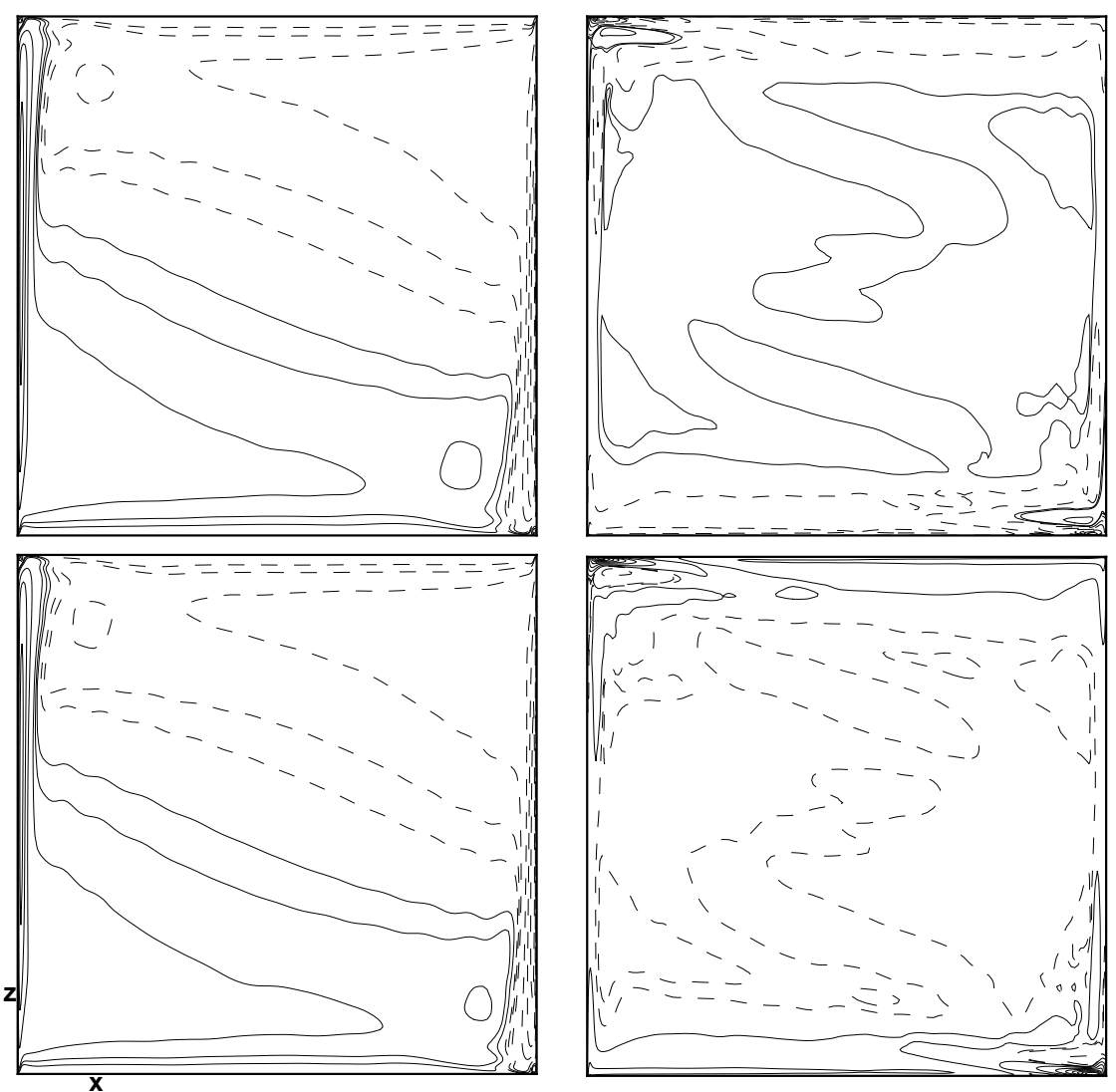

Figure 12: DNS results of $\langle w\rangle$ velocity (left) and $\langle v\rangle$ velocity (right) in the vertical planes at $y=0.02$ (top) and $y=0.30$ (bottom). 

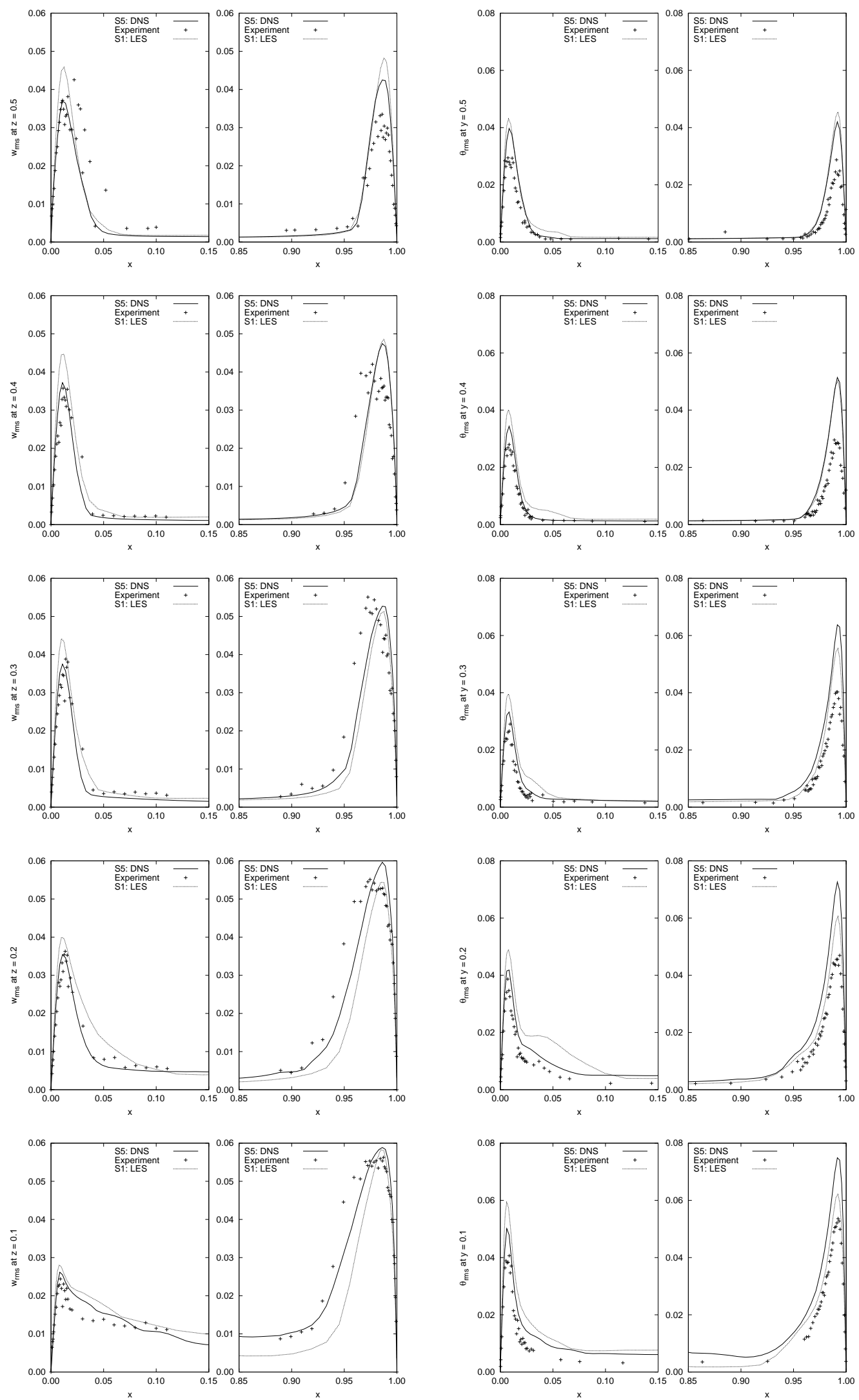

Figure 13: (a) $w_{r m s}$ and $\theta_{r m s}$ in the mid-depth vertical plane at $z=0.5,0.4,0.3,0.2$ and 0.1 (from top to bottom). 

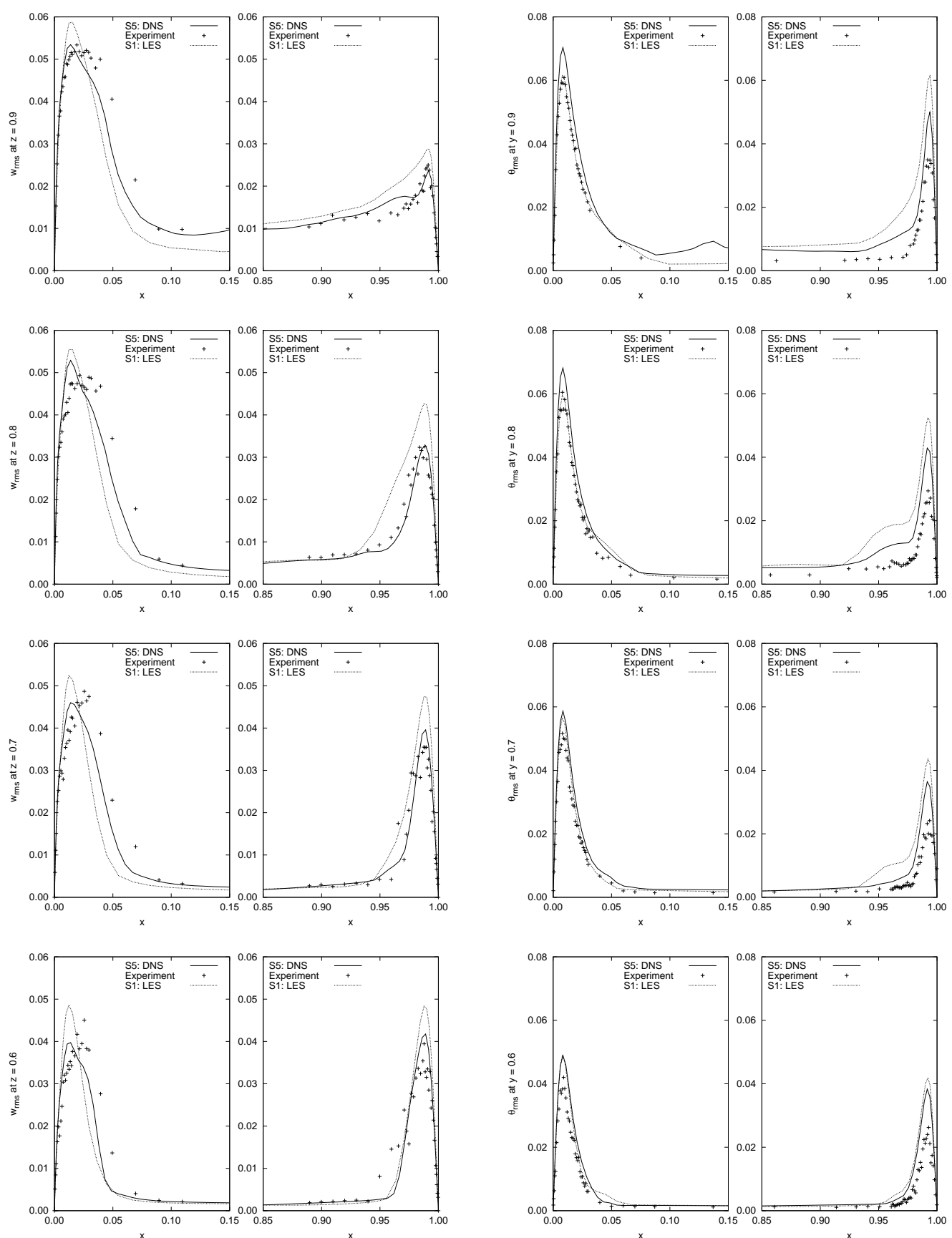

Figure 13: (b) $w_{r m s}$ and $\theta_{r m s}$ in the mid-depth vertical plane at $z=0.9,0.8,0.7$ and 0.6 (from top to bottom). 